\title{
BEHAVIOR OF HOLOMORPHIC FUNCTIONS IN COMPLEX TANGENTIAL DIRECTIONS IN A DOMAIN OF FINITE TYPE IN $\mathbb{C}^{n}$
}

\author{
Sandrine Grellier
}

\begin{abstract}
Let $\Omega$ be a damain in $\mathbb{C}^{n}$. It is known that a holomorphic function on $\Omega$ behaves better in complex tangential directions. When $\Omega$ is of finite type, the best possible improvement is quantified at each point by the distance to the boundary in the complex tangential directions (see the papers on the geometry of finite type domains of Catlin, Nagel-Stein and Wainger for precise definition). We show that this improvement is characteristic: for a holomorphic function, a regularity in complex tangential directions implies the corresponding regularity in all directions. We give a pointwise inequality in both directions between the gradients and the complex tangential gradients. We characterize Besov, Sobolev and Lipschitz spaces of holomorphic functions defined on $\Omega$ by the behavior of complex tangential derivatives.
\end{abstract}

\section{Introduction and Results}

Let $\Omega$ be a bounded $\mathcal{C}^{\infty}$-domain in $\mathbb{C}^{n}$. It is well known that a holomorphic function on $\Omega$ behaves twice as well in complex tangential directions: this result follows from the existence of a polydisc whose size, in the complex tangential directions, is proportional to the square root of the distance to the boundary $\partial \Omega$ (see $[\mathrm{G} \& \mathrm{~S}]$ and $[\mathrm{Kr} \mathbf{r}]$ for example). It is also well known that the converse is true when $\Omega$ satisfies (see $[\mathbf{H}$, [R\&S]):

(P) the tangent space is generated by the Lie brackets of real and imaginary parts of complex tangent vectors.

When $\Omega$ is of finite type (see $[\mathbf{K}]$ and $[\mathbf{B} \& \mathbf{G}]$ ), the improvement of the behavior of holomorphic functions in complex tangential directions is better. It follows from the existence of a polydisc whose size is larger in complex tangential directions and depends on the flatness of the boundary. In this case, there is also some kind of converse results: a regularity 
in complex tangential directions implies a corresponding regularity in all directions. In $[\mathrm{Kr} 2]$ and $[\mathrm{P}]$, some converse results follow from real variable methods on $\partial \Omega$, using the Campbell Haussdorff Formula for instance, while results in this direction may be considered as a special case of the deep theory of $[\mathrm{H}]$ and [R\&S].

Our method, which is clcmentary, is different from theirs and follows from the first proof of Stein on Lipschitz estimates (see [G\&S]). We give pointwise estimates between gradients and tangential gradients of holomorphic functions in both directions applying Cauchy Formula on the polydiscs inside $\Omega$. These estimates generalize to domains of finite type the results of $[G 1]$ which deal with domains satisfying the (P) property.

Let us define the following.

Writc $\Omega=\{r<0\}$, where $r$ is a $\mathcal{C}^{\infty}$ function satisfying

$$
d r \neq 0 \text { on } \partial \Omega=\{r=0\} .
$$

Define the complex tangential gradient of ordor $k$ of $u,\left\{\nabla_{7}^{k} u\right\}$, as follows.

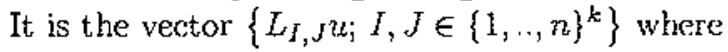

$$
L_{2, j}=\frac{\partial r}{\partial z_{i}} \frac{\partial}{\partial z_{j}}-\frac{\partial r}{\partial z_{j}} \frac{\partial}{\partial z_{i}} ; \quad i, j \in\{1, \ldots, n\}
$$

and $L_{i, J}=L_{i_{1}, j_{1}} \ldots L_{i_{k}, j_{k}}$ when $I=\left(i_{1}, \ldots, i_{k}\right), J=\left(j_{1}, \ldots, j_{k}\right)$.

Denote by $\delta($.$) the distance to the boundary of \Omega$. Assume that $\Omega$ is of finite type $m \in \mathbb{N}$. Following [NSW] or $[\mathrm{C}]$, we use a function $\tau()=$. $\tau(., c \delta()$.$) giving the size of the biggest ball inside \Omega$ in the complex tangential directions. This function satisfies $c \delta^{1 / 2}(.) \leq \tau(.) \leq C \delta(.)^{1 / m}$.

Roughly speaking, we are going to show that, for a holomorphic function $g$ defined on $\Omega$, a size estimate on $\left\{\delta^{l} \nabla^{l} g\right\}$ implies the same size estimate on $\left\{\tau^{k} \nabla_{T}^{k} g\right\}$ and aiso that the converse is true. The first part of our work is to majorize $\tau^{k}\left|\nabla_{T}^{k} g\right|$ by the mean-value of $|g|$ on a polydisc adapted to the geometry of $\Omega$. It is what we call the direct estimates. Such an cstimate is implicit in some works but is not explicitely written (see $[\mathrm{B}],[\mathrm{C} \& \mathrm{~K}]$ and $[\mathrm{Kr} 3]$ ). The main part of our work is to show the converse: $\delta^{k}\left|\nabla^{k} g\right|$ is majorized by the mean-value of $\tau^{k}\left|\nabla_{7}^{k} g\right|$, disregarding some remaining terms. It is what we call the converse estimates. Compared with the usual mean-value property of holomorphic functions, these pointwise estimates show that $\nabla_{T}^{k} g$ behaves at least as a complete gradient of order $k / m$ and at most as a complete gradient of order $k / 2$ (using the property that $\left.c \delta(.)^{1 / 2} \leq \tau(.) \leq C \delta(.)^{1 / m}\right)$. 
Using these pointwise inequalities, we characterize Besov, Sobolev and Lipschitz spaces of holomorphic functions on $\Omega$ by the behavior of complex tangential derivatives.

Let us notice that, when $n>2$, we should distinguish each complex tangential direction in order to obtain the optimal result; then, we would be able to measure the exact improvement of the behavior of holomorphic functions in each direction. But up to now, we do not know in which amount of generality one can construct apropriate polydises.

We use the following mean-value operator

$$
\operatorname{Mean}^{Q(z)}(u)=\frac{1}{|Q(z)|} \int_{Q(z)}|u(\zeta)| d V(\zeta) \quad z \in \Omega \cap U
$$

where $U$ is a neighborhood of $\partial \Omega, Q(z)$ is the biholomorphic image of a polydisc $R(z)$ centered at $z$, whose size is $c \delta(z)$ in the complex normal direction and $\tau(z)$ in the complex tangential directions; $c$ will be chosen so that, in particular, $Q(z) \subset \Omega$.

Now, let us state our results. We begin with the direct estimates.

\section{Theorem A.}

Let $\Omega$ be a $\mathcal{C}^{\infty}$-domain of finite type $m$ in $\mathbb{C}^{n}$. Let $k, l \in \mathbb{N}, p>0$. For each $z_{0} \in \partial \Omega$, there exist a neighborhood $V\left(z_{0}\right)$ and a constant $C$ such that, for every holomorphic function $g$ in $\Omega$ and every $z \in V\left(z_{0}\right) \cap \Omega$

(1) $\tau(z)^{k p}\left|\nabla_{T}^{k} g(z)\right|^{p} \leq C M e a n^{Q(z)}\left(|g|^{p}\right)$.

(2) $\delta(z)^{i p} \tau(z)^{k p}\left|\nabla^{l} \nabla_{T}^{k} g(z)\right|^{p} \leq C M \operatorname{Man} Q(z)\left(\delta^{i p} \sum_{j=1}^{i}\left|\nabla^{j} g\right|^{p}\right)$.

Also, we obtain converse estimates which allow us to cstimate a complete gradient by a tangential one, disregarding some remaining terms.

\section{Theorem B.}

Let $\Omega$ be a $\mathcal{C}^{\infty}$-domain of finite type $m$ in $\mathbb{C}^{n}$. Let $k \in \mathbb{N}$ and $p>0$. For each $z_{0} \in \partial \Omega$, there exist a neighborhood $V\left(z_{0}\right)$ and a constant $C$ such that:

for each $\varepsilon>0$, there exists a constant $C(\varepsilon)$ such that, for every holomorphic function $g$ in $\Omega$ and every $z \in V\left(z_{0}\right) \cap \Omega$

$$
\delta(z)^{k p}\left|\nabla^{k} g(z)\right|^{p} \leq C M \operatorname{ean}^{Q(z)}\left(C(\varepsilon) \tau^{k p}\left|\nabla_{r}^{k} g\right|^{p}+\operatorname{Rest}^{k}(\varepsilon)^{p}\right)
$$


where

$$
\operatorname{Rest}^{k}(\varepsilon)=\left(\sum_{1 \leq r+j \leq k(m-1)} \mathcal{O}_{r, j}^{k}(\varepsilon)\left|\nabla_{T}^{r} \nabla^{j} g\right|\right)
$$

and

$$
\mathcal{O}_{r, j}^{k}(\varepsilon)(z) \leq\left\{\begin{array}{l}
C(\varepsilon) \delta^{j+1}(z) \tau^{r}(z)+C \varepsilon \delta^{j}(z) \tau^{r}(z) \\
C(\varepsilon) \tau^{k}(z) \text { when } m j+r-k<0
\end{array}\right.
$$

Remark. The main terms in the previous two estimates are homogeneous in the following sense: each derivative of order $r$ in the complex tangential directions appears with a factor $\tau^{r}$ and each one in the other directions with a factor $\delta^{r}$. In the remaining term Rest ${ }^{k}(\varepsilon)$, cach derivative appears with a smaller factor.

By the mean-value property and Theorem A, we can majorize Rest ${ }^{k}(\varepsilon)$ by a small constant times $M e a n^{Q(z)}\left(|g|^{p}\right)$. However, for technical reasons, we will need this complicated form of $\operatorname{Rest}^{k}(\varepsilon)$ (in order to be ablc to apply Hardy inequalities for example).

Before giving the applications of the previous theorems, let us give the following result.

\section{Auxiliary Theorem.}

Let $\Omega$ be a bounded $\mathcal{C}^{\infty}$-domain of finite type $m$ in $\mathbb{C}^{n}$. There exists a compact set $K \subset \Omega$ such that, for every $p>0$, every $k \in \mathbb{N}$ and $q, t \in \mathbb{B}$ with $q+\frac{t}{m}>-1$ and $t \geq 0$,

$$
\int_{\Omega}|g|^{p} \delta^{q} \tau^{t} d V \text { and } \int_{\Omega}\left|\nabla^{k} g\right|^{p} \delta^{q+p k} \tau^{t} d V
$$

are equivalent, modulo an error of $\int_{K}|g|^{p} d V$, for $g$ holomorphic in $\Omega$.

In the same way, for every $k \in \mathbb{N}$ and every $q, t \in \mathbb{R}$ with $q+\frac{t}{m}>0$ and $t \geq 0$,

$$
\sup _{\Omega}\left\{|g| \delta^{q} \tau^{t}\right\} \text { and } \sup _{\Omega}\left\{\left|\nabla^{k} g\right| \delta^{q+p k} \tau^{t}\right\}
$$

are equivalent, modulo an error of $\sup _{K}|g|$, for $g$ holomorphic in $\Omega$.

As in [G1], we shall deduce from the estimates of Theorem $A$ and $B$ a characterization of Besov, Sobolev, and Lipschitz spaces in terms of complex tangential derivatives.

The first theorem is an $L^{p}$-application of the previous estirnates and will allow us to obtain a characterization of Besov and Sobolev spaces in terms of complex tangential derivatives. 


\section{Theorem C.}

Let $\Omega$ be a bounded $\mathcal{C}^{\infty}$-domain of finite type $m$ in $\mathbb{C}^{n}$. There exists a compact set $K \subset \Omega$ such that, for every $p>0 q, t \in \mathbb{R}$ and $k, l, r \in \mathbb{N}$ with $q+p r+\frac{t}{m}>-1, q+p l+\frac{p k+t}{m}>-1$ and $t \geq 0$

$$
\begin{aligned}
& \int_{\Omega}\left|\nabla_{T}^{k} \nabla^{l} g\right|^{p} \delta^{q+p l} \tau^{k p+t} d V \\
& \int_{\Omega}\left|\nabla^{l} \nabla_{T}^{k} g\right|^{p} \delta^{g+p l} \tau^{k p+t} d V \text { and } \\
& \int_{\Omega}\left|\nabla^{r} g\right|^{p} \delta^{q+p r} \tau^{t} d V
\end{aligned}
$$

are equivalent, modulo an error of $\int_{K}|g|^{p} d V$, for g holomorphic in $\Omega$.

A second theorem may be considered as an $L^{\infty}$-application of Theorems $\mathrm{A}$ and $\mathrm{B}$ and will allow us to obtain a characterization of Lipschitz spaces in terms of complex tangential derivatives.

\section{Theorem D.}

Let $\Omega$ be a bounded $\mathcal{C}^{\infty}$-domain of fnite type in $\mathbb{C}^{n}$. There exists a compact set $K \subset \Omega$ such that, for every $q, t \in \mathbb{R}$, every $k, l, r \in \mathbb{N}$ with $q+l+\frac{k+t}{m}>0, t \geq 0$ and $q+r+\frac{t}{m}>0$,

$$
\begin{aligned}
& \sup _{\Omega}\left\{\left|\nabla_{T}^{k} \nabla^{l} g\right| \delta^{g+l} \tau^{k+\imath}\right\}, \\
& \sup _{\Omega}\left\{\left|\nabla^{i} \nabla_{T}^{k} g\right| \delta^{q+i} \tau^{k+t}\right\} \text { ond } \\
& \sup _{\Omega}\left\{\left|\nabla^{r} g\right| \delta^{\eta+r} \tau^{l}\right\}
\end{aligned}
$$

are equivalent, modulo an error of $\sup _{K}|g|$, for $g$ holomorphic in $\Omega$.

We use the following notations.

- $\mathcal{H}(\Omega)$ is the set of holomorphic functions on $\Omega$.

- For $\gamma>-1$ and $s \in \mathbb{N}, B_{s_{1} \gamma}^{p}$ is the Besov space of order $s$ and weight $\gamma$ defined by

$$
B_{s, \gamma}^{p}=\left\{g \in \mathcal{H}(\Omega) ; \int_{\Omega}\left|\nabla^{s} g\right|^{p} \delta^{\gamma} d V<\infty\right\}
$$

- For $\alpha>0, \Lambda_{\alpha}(\Omega)$ is the usual Lipschitz space on $\Omega$ (see $[\mathrm{Kr} 1]$ ). 
- For $s \geq 0$ and $p \geq 1, W^{s, p}(\Omega)$ is the Sobolev space defined as in [Gr] to be the space of functions $u$ satisfying

$$
\iint_{\Omega \times \Omega \Omega} \frac{|u(z)-u(\zeta)|^{p}}{|z-\zeta|^{2 n+s p}} d V(z) d V(\zeta)<\infty
$$

As usual, this definition is extended to negative value of $s$ by duality.

We recall (cf $[D]$ ) that,

$$
\begin{aligned}
& \Lambda_{\alpha}(\Omega) \cap \mathcal{H}(\Omega)= \\
& \quad=\left\{g \in \mathcal{H}(\Omega) ; \sup _{\Omega}\left\{\delta^{k-\alpha}\left|\nabla^{k} g\right|\right\}<\infty \text { for every integer } k>\alpha\right\} .
\end{aligned}
$$

Then, we obtain the following corollary.

Corollary A.

Let $\Omega$ be a bounded $\mathcal{C}^{\infty}$-domain of finite type in $\mathbb{C}^{n}$. Let $p>0, \gamma>-1$, $\alpha>0$. Then, for everyg holomorphic in $\Omega$,

$$
\begin{aligned}
& \text { (1) } g \in B_{s, \gamma}^{p} \Leftrightarrow \int_{\Omega}\left|\nabla_{T}^{k} g\right|^{p} \delta^{\gamma-p, s} T^{k p} d V<\infty \\
& \text { for } k \text { integer, } k>m\left(s-\frac{\gamma+1}{p}\right), \\
& \text { (2) } g \in \Lambda_{\alpha}(\Omega) \Leftrightarrow \sup _{\Omega}\left\{\tau^{k} \delta^{-\alpha}\left|\nabla_{T}^{k} g\right|\right\}<\infty . \\
& \quad \text { for } k \text { integer, } k>m \alpha>0 .
\end{aligned}
$$

It is known that, for $s \geq 0$, the Sobolev space $W^{s, D}(\Omega) \cap \mathcal{H}(\Omega)$ is equal to $B_{k, p(k-s)}^{p}(\Omega)$ when $p \geq 1$ and $k$ is an integer such that $p(k-s)>-1$ (see [Bo\&Si], appendix); when $s<0, W^{s, p}(\Omega) \cap \mathcal{H}(\Omega)$ is equal to $B_{0,-s p}^{p}(\Omega)$ (see $[\mathbf{L}]$ ). This gives, with the previous corollary, a characterization of $W^{s_{1} p}(\Omega) \cap \mathcal{H}(\Omega)$ in terms of complex tangential derivatives.

As in the case when $\Omega$ satisfies the (P) property (see $[\mathrm{G} 1]$ ), we can characterize the holomorphic functions $g$ such that $\nabla_{T}^{k} g \in W^{s, p}(\Omega)$ in terms of a Besov property.

\section{Theorem E.}

Let $\Omega$ be a bounded $\mathcal{C}^{\infty}$-domain of finite type and $s \geq 0$. There exist a compact set $K \subset \Omega$ and a constant $C>0$ such that, for every holomorphic function $g$ in $\Omega$,

$$
\int_{\Omega}\left|\nabla^{l} \nabla_{T}^{k} g\right|^{p} \delta^{p(l-s-k / m)} \tau^{k p} d V \leq C\left(\left\|\nabla_{T}^{k} g\right\|_{W^{s, p}(\Omega)}^{p}+\int_{K}|g|^{p} d V\right)
$$

for $l$ integer, $p(l-s)>-1$.

A sirnilar theorem is available for Lipschitz spaces. 


\section{Theorem $\mathbf{F}$.}

Let $\Omega$ be a bounded $\mathcal{C}^{\infty}$-domain of finite type and $\alpha>0$. There exist a compact set $K \subset \Omega$ and a constant $C>0$ such that, for every holomorphic function $g$ in $\Omega$,

$$
\sup _{\Omega}\left\{\left|\nabla^{l} \nabla_{T}^{k} g\right| \delta^{(l-\alpha-k / m)} \tau^{k}\right\} \leq C\left(\left\|\nabla_{T}^{k} g\right\|_{\Lambda_{\alpha}(\Omega)}+\sup _{K}\{|g|\}\right)
$$

for l integer, $l>\alpha$.

These two results give the key to the following corollary.

\section{Corollary B.}

Let $\Omega$ be a bounded $\mathcal{C}^{\infty}$-domain of finite type $m \in \mathbb{N}$, let $k \in \mathbb{N}$ and $s \geq 0$. Let $g$ be a holomorphic function on $\Omega$ such that $\nabla_{i}^{k} g \in W^{s, p}(\Omega)$ (resp. $\Lambda_{\alpha}(\Omega)$ ); then $g$ is in $W^{s+k / m, p}(\Omega)$ (resp. $\Lambda_{\alpha+k / m}(\Omega)$ ).

As a reciprocal result, we can prove that, for a holomorphic function $g$ and for $s \geq 0, g \in W^{s+k / 2, p}(\Omega)$ (resp. $g \in \Lambda_{c x+k / 2}(\Omega)$ ) implies that $\nabla_{T}^{k} g \in W^{s, p}(\Omega)$ (resp. $\nabla_{T}^{k} g \in \Lambda_{\alpha}(\Omega)$ ), which is the best result of this kind one can hope. For this result, we use the fact that ( $\operatorname{cf}[\mathrm{B} \circ \& \mathrm{Si}]$ ), for every $s \geq 0$, every integer $k$ such that $p(k-s)>-1$ and every function $u$ defined on $\Omega$ satisfying

$$
\int_{\Omega}\left|\nabla^{k} u\right|^{p} \delta^{p(k-s)} d V<\infty
$$

then $u \in W^{s, p}(\Omega)$.

When $k=1$, Corollary B can be deduced from the work of Rostchild. Stein (see [R\&S]) or from the work of Krantz for Lipschitz spaces (see [Kr2]).

Remarks. - The finite type hypothesis is not necessary to obtain direct estimates. As in [B], we can define a new function $\tau_{m}$ for cach $m \geq 2$, without any assumption on the type of $\Omega$, which allows us to define a now pseudo-metric on the level lines of $\Omega$ with the help of $\tau_{m}$. Then, the direct estimates are true with this new function for all $m \geq 2$.

- A counterexarnple. The conditions on the orders of derivatives $k, l, r$ in Theorems $C$ and $D$ are sharp.

For example, when $\Omega=\left\{z \in \mathbb{C}^{2} ;\left|z_{1}\right|^{2}+\left|z_{2}\right|^{1}<1\right\}$ which is of finite type 4 , let us define

$$
g(z)=z_{2} \log \left(1-z_{1}\right)
$$


on $\Omega$. Then it is easy to verify that $g \in \Lambda_{1 / 4}(\Omega) \cap \mathcal{H}(\Omega)$ (because $\left.|\nabla g(z)| \leq C \delta(z)^{-3 / 4}\right)$ although $|L g(z)|$ is not boinded by $\frac{\delta(z)^{3 / 4}}{\tau(z)}$.

The proofs of Theorems A and B will use the following new system of coordinates.

\section{Special Coordinates and Polydiscs}

Let $\Omega$ be as before. Let $z_{0} \in \partial \Omega$; as $d r\left(z_{0}\right) \neq 0$, we may assume that $\frac{\partial r}{\partial z_{1}} \neq 0$ in a neighborhood $V\left(z_{0}\right)$ of $z_{0}$. The following Lernma is a version of a result of Catlin (sce $[\mathbf{C}]$, and also $[\mathrm{F} \& \mathrm{~S}]$ ).

Lemma 1.1. For each $z \in V\left(z_{0}\right) \cap \Omega$, there exists a biholomorphic mapping $\Phi_{z}$ from $\mathbb{C}^{n}$ to itself such that the function $\varrho(\zeta)=r \circ \Phi_{z}(\zeta)$ is of the form

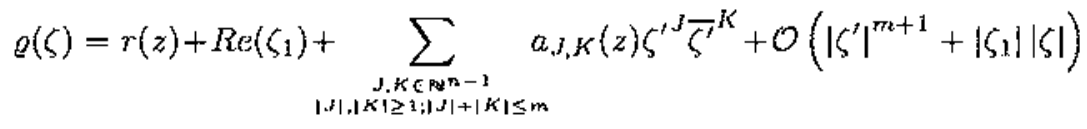

where $\zeta^{\prime}=\left(\zeta_{2}, \ldots, \zeta_{n}\right)$ and also

$$
\Phi_{z}(\zeta)=\left(z_{1}+d_{0}(z) \zeta_{1}+\sum_{|L|=1}^{m} d_{L}(z) \zeta^{\prime \prime}, z_{2}+\zeta_{2}, \ldots, z_{n}+\zeta_{n}\right)
$$

where $d_{0}(),. d_{L}(.) ;|L|=1, \ldots, m$ depend smoothly on $z$ and $d_{0}() \neq$.0 in $V\left(z_{0}\right)$.

Remark. This lemma allows us to estimate $\varrho$ and its derivatives: it gives that.

$$
\begin{gathered}
\frac{\partial^{J} \varrho}{\partial \zeta^{j}}(0)=0 \text { for } J \in \mathbb{N}^{n-1} \text { with } 1 \leq|J| \leq m, \quad \frac{\partial \varrho}{\partial \zeta_{1}}(0)=\frac{1}{2} \\
\frac{\partial^{|J|+|K|} \varrho}{\partial{\zeta^{\prime}}^{\prime} \partial \bar{\zeta}^{K}}(0)=a_{J, K}(z) \text { for } J, K \in \mathbb{N}^{n-1},|J|,|K| \geq I ;|J|+|K| \leq m .
\end{gathered}
$$

Proof: We are going to apply the same argument as in [C] to construct the function $\Phi_{z}$ by induction on $m$.

For each $z \in V\left(z_{0}\right)$, we set

$$
\Phi^{1}(\zeta)=\left(z_{1}+\left(\frac{\partial r}{\partial z_{1}}(z)\right)^{-1}\left(\frac{\zeta_{1}}{2}-\sum_{j=2}^{n} \frac{\partial r}{\partial z_{j}}(z) \zeta_{j}\right), z_{2}+\zeta_{2}, . ., z_{n}+\zeta_{n}\right) .
$$


then $\varrho_{2}(\zeta)=\operatorname{ro\Phi ^{1}}(\zeta)=\operatorname{Re}\left(\zeta_{1}\right)+\mathcal{O}\left(\left.\zeta\right|^{2}\right)$.

Assume, now, that we have constructed a function $\Phi^{l-1}: \mathbb{C}^{n} \rightarrow \mathbb{C}^{n}$ such that $\varrho_{l}=r \circ \Phi^{l-1}$ satisfies

$(*)$

$$
\varrho_{l}(\zeta)=r(z)+\operatorname{Re}\left(\zeta_{1}\right)+\sum_{\substack{J, K \in \mathbb{N} n-1 \\ \lambda|,| K|\geq 1 ;|,|\cdots| K \mid<1}} a_{J, K}(z) \zeta^{\prime j} \bar{\zeta}^{K}+\mathcal{O}\left(\left|\zeta^{\prime}\right|^{l}+\left|\zeta_{1}\right||\zeta|\right) .
$$

If we define $\Phi^{l}=\Phi^{l-1} \circ \phi^{l}$ where

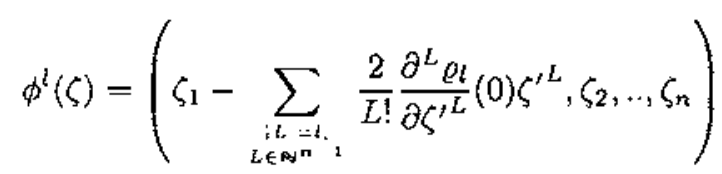

then $\varrho_{t+1}=r \circ \Phi^{l}$ satisfies tho analog of $(*)$ with $l$ replaced by $l+1$.

Thus, if we set $\Phi_{z}=\Phi^{m}$, we have finished the proof of the lemrna.

Now we are able to define the polydisc around $z$, as in $[\mathrm{C}]$.

We set $A_{l}(z)=\max \left\{\left|a_{J, K}(z)\right| ;|J|+|K|=l\right\}$; then, as $\Omega$ is of finite type $m$, there exists $l \in\{2, \ldots, m\}$ such that $A_{l}(z) \neq 0$ for $z \in \partial \Omega$ and furthermore, for $z$ in a sufficiently small neighborhood $V\left(z_{0}\right)$. Then, $\tau(z, \delta)=\min \left\{\left(\frac{\delta}{A_{i}(z)}\right)^{1 / l}, \quad l=2, \ldots, m\right\}$ is well defined. Let us define now

$$
Q_{\delta}(z)=\Phi_{z}\left(R_{\delta}(z)\right)=\Phi_{z}\left(\left\{\zeta \in \mathbb{C}^{n} ; \quad\left|\zeta_{1}\right| \leq \delta, \quad\left|\zeta^{\prime}\right| \leq \tau(z, \delta)\right\}\right) .
$$

One can verify that the properties satisfied by $\tau(z, \delta)$ and $Q_{\delta}(z)$ when $n=2$ are still available in the general case.

We give some of them.

1 - There exist some constants $c, C>0$ such that, for every $z \in V\left(z_{0}\right)$ and every $0<\delta<1$, we have

$$
c \delta^{1 / 2} \leq \tau(z, \delta) \leq C \delta^{1 / m} .
$$

2 - For cvery $\delta$ sufficiently small and every $z \in Q_{\delta}\left(z^{\prime}\right), \tau(z, \delta) \simeq$ $\tau\left(z^{\prime}, \delta\right)$.

3 - There exists a constant $C>0$ such that, if $z \in Q_{b}\left(z^{\prime}\right)$ then $Q_{\delta}(z) \subset Q_{C \delta}\left(z^{\prime}\right)$ and $Q_{\delta}\left(z^{\prime}\right) \subset Q_{C \delta}(z)$.

4 - If $\delta^{\prime}<\delta^{\prime \prime}$ then $\left(\frac{\delta^{\prime}}{\delta^{\prime \prime}}\right)^{1 / 2} \tau\left(z, \delta^{\prime \prime}\right) \leq \tau\left(z, \delta^{\prime}\right) \leq\left(\frac{\delta^{\prime}}{\delta^{\prime \prime}}\right)^{1 / m} \tau\left(z, \delta^{n}\right)$.

We conclude that, obviously, there exists a constant $c$ such that, for each $z \in V\left(z_{0}\right), Q_{c \delta(z)}(z) \subset \Omega$. We will write $Q(z)=Q_{c \delta(z)}(z)$ and $R(z)=R_{c \delta(z)}(z)$. 
5 . In addition, for every $\zeta \in Q(z), \tau(\zeta) \simeq \tau(z)$

(where $\tau(z)$ stands for $\tau(z, c \delta(z))$ ).

6 - By definition of $\tau\left(\right.$.), for each $z \in V\left(z_{0}\right)$, there exist $J, K \in \mathbb{N}^{n-1}$ with $|J|,|K| \geq 1,|J|+|K| \leq m$ such that $\left|a_{J, K}(z)\right| \tau(z)^{|J|+|K|}=\delta(z)$, while for every $(J, K)$, we have $\left|a_{J, K}(z)\right| \tau(z)^{|J|+|K|} \leq \delta(z)$. So, for each $z \in V\left(z_{0}\right)$, the following holds:

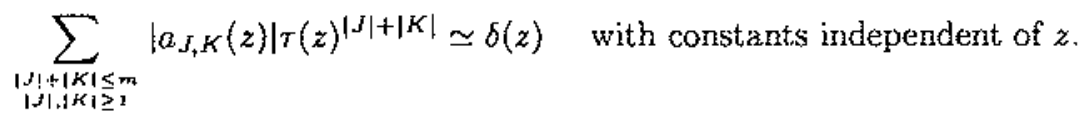

Remark. In the work of Nagel, Stein and Wainger (see [NSW]), there is an equivalent definition of the function $\tau$ which is intrisic. Explicitely, these authors construct an intrisic function $\Lambda(z, t)$ for any $z$ in $\Omega$ near $\partial \Omega$ and any $t>0$ sufficiently small such that $\Lambda(z, \tau(z, \delta)) \simeq \delta$.

\section{Direct Estimates: proof of Theorem $\mathbf{A}$}

We are going to prove Theorem A. Namely

\section{Theorem A.}

Let $\Omega$ be $a \mathcal{C}^{\infty}$-domain of finite type $m$ in $\mathbb{C}^{n}$. Let $k, l \in \mathbb{N}, p>0$. For each $z_{0} \in \partial \Omega$, there exist a neighborhood $V\left(z_{0}\right)$ and a constant $C$ such that, for every holomorphic function $g$ in $\Omega$ and every $z \in V\left(z_{0}\right) \cap \Omega$

(1) $\tau(z)^{k p}\left|\nabla_{T}^{k} g(z)\right|^{p} \leq C \operatorname{Mean}^{Q(z)}\left(|g|^{p}\right)$.

$$
\delta(z)^{l p} \tau(z)^{k_{p}}\left|\nabla^{l} \nabla_{T}^{k} g(z)\right|^{p} \leq C M \operatorname{ean}^{Q(z)}\left(\delta^{l p} \sum_{j=1}^{l}\left|\nabla^{j} g\right|^{p}\right) .
$$

To simplify, we are going to prove this theorem in $\mathbb{C}^{2}$. Given $z \in V\left(z_{0}\right)$ and $g$ holomorphic in $\Omega$, we set $f(\zeta)=g \circ \Phi_{z}(\zeta)$. Then $f$ is holomorphic in $\Omega_{z}=\Phi_{z}^{-1}(\Omega)$. We denote by $L^{\prime}=\frac{\partial \rho}{\partial \zeta_{2}} \frac{\partial}{\partial \zeta_{1}}-\frac{\partial \ell}{\partial \zeta_{2}} \frac{\partial}{\partial \zeta_{2}}$ a holonorphic tangent vector field.

In order to prove Theorem A, we are going to apply Cauchy Formula to $f$.

For cvery $j, r \in \mathbb{N}$ and every $p>0$

$$
\left|\frac{\partial^{i+r} f}{\partial \zeta_{1}^{j} \partial \zeta_{2}^{r}}(0)\right|^{p} \leq \frac{C}{\delta(z)^{2+p j} \tau(z)^{2+p r}} \int_{\left|\zeta_{1}\right| \leq c \delta(z) ;\left|\zeta_{2}\right| \leq \tau(z)}|f(\zeta)|^{p} d V(\zeta) .
$$

We see that the domain of integration is $R(z)$. In order to conclude, it is sufficient to remark, before changing again the system of coordinates, that $L^{\prime k} f(0)$ is almost equal to $\left(\frac{1}{2}\right)^{k} \frac{\partial^{k} f}{\partial \alpha_{2}^{k}}(0)$. In fact, we have the following lemma. 
Lemma 2.1. Let $k \in \mathbb{N}^{*}$. For every $\mathcal{C}^{\infty}$-function $u$, we have

$$
L^{\prime k} u(0)=\left(-\frac{1}{2}\right)^{k} \frac{\partial^{k} u}{\partial \zeta_{2}^{k}}(0)+\sum_{1 \leq m j+r \leq k-1} \mathcal{O}\left(\frac{\partial^{j+r} u}{\partial \zeta_{1}^{j} \partial \zeta_{2}^{r}}(0)\right)
$$

where the $\mathcal{O}$ are uniform.

Proof: We can show by induction on $k \in \mathbb{N}$ that there exist some constants $c_{j, r}, I \leq j+\tau \leq k$, such that

$$
L^{\prime k}=\sum_{1 \leq j+r \leq k} \sum_{E_{k, j, r}} c_{j_{r} r}\left(\prod_{i=1}^{k} \frac{\partial^{m_{i}+n_{i}} \varrho}{\partial \zeta_{1}^{m_{i}} \partial \zeta_{2}^{n_{i}}}\right) \frac{\partial^{3+r}}{\partial \zeta_{1}^{j} \partial \zeta_{2}^{r}}
$$

where $E_{k, 3, r}$ denotes the set of couples $\left(n_{i}, n_{i}\right), i=1, \ldots, k$, which are in lexicographical order and satisfy $\sum_{i=1}^{k} m_{i}=k-j, \sum_{i=1}^{k} n_{i}=k-r$ with $m_{i}+n_{i} \geq 1$. Furthermore, there exists at least one integer $i_{0} \in\{1, \ldots, k\}$ such that $\left(m_{i_{0}}, n_{i_{0}}\right)=(0,1)$ or $(1,0)$. So

$$
L^{\prime k} u(0)=\sum_{1 \leq j+r \leq k} \sum_{E_{k_{1}, \cdot}} c_{j, r}\left(\prod_{i=1}^{k} \frac{\partial^{m_{i}+n_{i}} \varrho}{\partial \zeta_{1}^{m_{i}} \partial \zeta_{2}^{n_{2}}}(0)\right) \frac{\partial^{j+r} u}{\partial \zeta_{1}^{j} \partial \zeta_{2}^{r}}(0)
$$

Since $\varrho$ is a $C^{\infty}$-function, each coefficient of this sum is uniformly bounded. So, it suffices to show that the coefficient of $\frac{\partial^{j+r} u}{\partial \zeta_{1}^{j} \partial \zeta_{2}^{r}}(0)$ is cqual to zero except when $m j+r \leq k-1$. By assumption, we have $\frac{\partial^{n} \rho}{\partial \zeta_{2}^{n}}(0)=0$ for $1 \leq n \leq m$. So, the only non-zero coefficients come from a subset of $E_{k, j, r}$ which is equal to the set of couples $\left(m_{i}, n_{i}\right)$ such that, either $m_{i}>0$ or $m_{i}=0$ and $n_{i}>m$.

Let us denote by $J$ the number of $m_{i}$ which are equal to zero. We have $m_{1}=m_{2}=\ldots=m_{J}=0$. By assumption, $k-j=\sum_{i=j+1}^{k} m_{i} \geq k-J$ and $k-r=\sum_{i=1}^{J} n_{i}+\sum_{i=J+1}^{k} n_{i}>m J$.

So, $k-r>m J \geq m j$. The result follows.

Then, the first part of Theorem A follows from Lernma 2.1 and from Cauchy Formula. To prove the second part, it is sufficient to apply the previous estimate to the derivatives of $g$ and to use the following easy lemma which is left to the reader. 
Lemma 2.2. For every $l \in \mathbb{N}, k \in \mathbb{N}^{\star}$ and $u \in \mathcal{C}^{\infty}(\Omega)$, we have

$$
\begin{aligned}
& \left|\nabla^{i} \nabla_{T}^{k} u\right| \leq\left|\nabla_{T}^{k} \nabla^{i} u\right|+\mathcal{O}\left(\sum_{\substack{1 \leq j \leq 1 \\
0 \leq r \leq k-1}}\left|\nabla_{T}^{r} \nabla^{j} u\right|\right) \\
& \left|\nabla_{T}^{k} \nabla^{l} u\right| \leq\left|\nabla^{l} \nabla_{T}^{k} u\right|+\mathcal{O}\left(\sum_{\substack{1 \leq j \leq d \\
0 \leq r \leq k-1}}\left|\nabla_{T}^{r} \nabla^{j} u\right|\right)
\end{aligned}
$$

where the $\mathcal{O}$ are uniform on $\Omega$.

\section{Converse Estimates: proof of Theorem B}

We are going to prove Theorem B. More precisely, we are going to prove the following.

\section{Theorem B.}

Let $\Omega$ be a $\mathcal{C}^{\infty}$-domain of finite type $m$ in $\mathbb{C}^{n_{2}}$. Let $k \in \mathbb{N}$ and $p>0$. For each $z_{0} \in \partial \Omega$, there exist a neighborhood $V\left(z_{0}\right)$ and a constant $C$ such that:

for each $\varepsilon>0$, there exists a constant $C(\varepsilon)$ such that, for every holomorphic function $g$ in $\Omega$ and every $z \in V\left(z_{0}\right) \cap \Omega$

$$
\delta(z)^{k p}\left|\nabla^{k} g(z)\right|^{p} \leq C \operatorname{Mean}^{Q(z)}\left(C(\varepsilon) \tau^{k p}\left|\nabla_{T}^{k} g\right|^{p}+\operatorname{Rest}^{k}(\varepsilon)^{p}\right)
$$

where

$$
\operatorname{Rest}^{k}(\varepsilon)=\left(\sum_{1 \leq r+j \leq k(m-1)} \mathcal{O}_{r, j}^{k}(\varepsilon)\left|\nabla_{T}^{r} \nabla^{j} g\right|\right)
$$

and

$$
\mathcal{O}_{r, j}^{k}(\varepsilon)(z)=\left\{\begin{array}{l}
C(\varepsilon) \delta^{j+1}(z) \tau^{r}(z)+C \varepsilon \delta^{j}(z) \tau^{r}(z) \text { if } \delta^{j}(z) \tau^{r}(z) \leq c \tau^{k}(z) \\
C(\varepsilon) \delta^{j}(z) \tau^{r+1}(z) \text { if } c r^{k}(z) \leq \delta^{j}(z) \tau^{r}(z) \leq C \tau^{k-1}(z) \\
C(\varepsilon) \tau^{k}(z) \text { otherwise. }
\end{array}\right.
$$

Let us first give a result by Ahern and Bruna. We give the following definition.

Definition. Let $\Omega$ be an open set in $\mathbb{C}^{n}$. Let $K=\left(k_{1}, \ldots, k_{n}\right)$ be a multi-index of integers. A function $F \in \mathcal{C}^{\infty}(\Omega)$ is called $(\mathrm{AB})_{K}$ if $\frac{\partial^{k_{j}} \bar{k}}{\partial{\overline{\zeta_{j}}}^{k_{j}}}=0$ for $j=1$ to $n$.

To simplify, we will assume that $K$ is fixed in the following and we will write $(A B)$ instead of $(A B)_{K}$.

For every $\zeta \in \mathbb{C}, r>0$, we denote by $D(\zeta, r)$ the disc $\left\{z \in \mathbb{C}_{i}|z-\zeta| \leq\right.$ $r\}$. Then, we have the following lemma (see $[\mathbf{A} \& \mathbf{B}])$. 
Lemma 3.1. For every $L, M \in \mathbb{N}^{n}, 0<p<\infty$, there exists a constant $C$ such that, for every $(A B)$ function $F$ in $\Omega$, every $\zeta=\left(\zeta_{1}, . ., \zeta_{n}\right) \in$ $\Omega$ and every $r=\left(r_{1}, . ., T_{n}\right) \in\left(\mid 0,+\infty[)^{n}\right.$ with $D\left(\zeta_{1}, r_{1}\right) \times \ldots \times D\left(\zeta_{n}, r_{n}\right) \subset$ $\Omega$, we have

$$
\left|\frac{\partial^{|L|+|M|} F}{\partial \bar{\zeta}^{L} \partial \zeta^{M}}(\zeta)\right|^{p} \leq \frac{C}{\prod_{j=1}^{n} r_{j}^{p\left(L_{j}+M_{j}\right)+2}} \int_{D\left(\zeta_{1}, r_{1}\right) \times \ldots \times D\left(\zeta_{\left.x_{t}, r_{n}\right)}\right.}|F|^{p} d V .
$$

Let us go back to the proof of Theorem B. As in the preceding paragraph, we will give the proof of this theorcm in $\mathbb{C}^{2}$.

\section{A particular case.}

First, we are going to give the main ideas of the proof by considering the case of gradients of order 1 , that is $k=1$. For $z \in V\left(z_{0}\right)$, we set $f(\zeta)=g\left(\Phi_{z}(\zeta)\right)$. We dernote by $L^{\prime}=\frac{\partial p}{\partial \zeta_{2}} \frac{\partial g}{\partial \zeta_{1}}-\frac{\partial p}{\partial \zeta_{1}} \frac{\partial}{\partial \zeta_{2}}$ a complex tangential vector field in $\Phi_{z}^{-1}(\Omega)$.

As $\frac{\partial \rho}{\partial \zeta_{1}}(0)=\frac{1}{2}, \quad \frac{\partial g}{\partial \zeta_{2}}(0)=0, \frac{\partial}{\partial \zeta_{2}}$ is locally a transwerse vector ficld and it suffices to estimate $\left(\frac{\delta(z)}{\tau(z)}\right)^{p}\left|\frac{\partial f}{\partial \zeta_{1}}(0)\right|^{p}$ in terms of $\left|L^{\prime} f\right|^{p}$ in order to estimate $\left(\frac{\delta(z)}{\tau(z)}\right)^{p}\left|\nabla_{g}(z)\right|^{p}$ in terms of $\left|\nabla_{T} g\right|^{p}$. By the Taylor expansion of $\varrho$ given in Lemma 1.1 , we have, on $R(z)$,

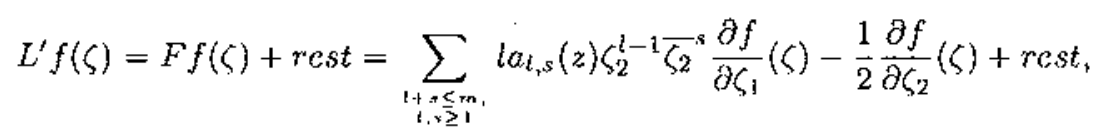

where $F f$ is an $(\mathrm{AB})$ function, since $f$ is holomorphic, which satisfies in particular

$$
\tau(z)^{p(j+k)}\left|\frac{\partial^{j+k} F f}{\partial \zeta_{2}^{j} \partial{\overline{\zeta_{2}}}^{k}}(0)\right|^{p} \leq C M o y y^{\prime \prime(z)}\left(|F f|^{p}\right) .
$$

As $\sum\left|a_{l, s}(z)\right| \tau(z)^{l+s} \simeq \delta(z)$ by property 6 , the simpkst mothod should be to estimate.

$$
\left|a_{l, s}(z)\right|^{p} \tau(z)^{p(l+s-1)}\left|\frac{\partial f}{\partial \zeta_{1}}(0)\right|^{p}
$$

for each $l, s$, in terms of the moan-value of $|F f|^{p}$ in order to estimate

$$
\left(\frac{\delta(z)}{\tau(z)}\right)^{p}\left|\frac{\partial f}{\partial \zeta_{1}}(0)\right|^{p}
$$

in terms of the mean-value of $\left|L^{\prime} f\right|^{p}$, disregarding a rest. 
Each $a_{l, s}(z) \frac{\partial f}{\partial \zeta_{1}}(0)$ appenars, up to a constant, as a component of a

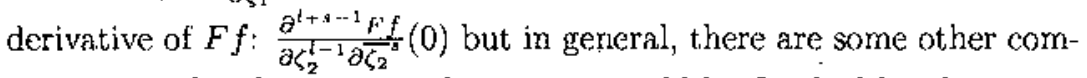
ponents in this derivative, otherwise it would be finished by the meanvalue property $(*)$. So, we have to write $\frac{\partial^{t+s^{* 1} F_{f}}}{\partial \zeta_{2}^{i-1} \partial \overline{\zeta_{2}}}(0)$ as the sum of $a_{l, s}(z) \frac{\partial f}{\partial \zeta_{1}}(0)$ and a rest, using an induction method. Let us look at this method when $m=3$. We have

$$
\frac{\partial F f}{\partial \widetilde{\zeta_{2}}}(0)=a_{1,1}(z) \frac{\partial f}{\partial \zeta_{1}}(0) \text { and } \frac{\partial^{2} F f}{\partial{\overline{\zeta_{2}}}^{2}}(0)=a_{1,2}(z) \frac{\partial f}{\partial \zeta_{1}}(0) .
$$

So, wc obtain an estimate on

$$
\left|a_{1,1}(z)\right|^{p} \tau(z)^{p}\left|\frac{\partial f}{\partial \zeta_{1}}(0)\right|^{p}
$$

and on

$$
\left|a_{1,2}(z)\right|^{p} \tau(z)^{2 p}\left|\frac{\partial f}{\partial \zeta_{1}}(0)\right|^{p}
$$

in terms of the mean-value of $|F f|^{p}$ on $R(z)$. It remains to consider the term $a_{2,1}(z) \frac{\partial f}{\partial \zeta_{2}}(0)$. We have

$$
\frac{\partial^{2} F f}{\partial \overline{\zeta_{2}} \partial \zeta_{2}}(0)=a_{2,1}(z) \frac{\partial f}{\partial \zeta_{1}}(0)+a_{1,1}(z) \frac{\partial^{2} f}{\partial \zeta_{1} \partial \zeta_{2}}(0) .
$$

But now, we can assurnc that $\left|a_{1,1}(z)\right| \tau^{2}(z)$ is very small compared with $\delta(z)$, otherwise we can use the preceding estimate. This allows to see that the corresponding term in $\frac{\left.\partial^{2} F\right\}}{\partial \zeta_{S_{2}} \partial \zeta_{2}}(0)$ is a rest. We will not go into all details as this will be done in the general case.

The general case (in $\mathbb{C}^{2}$ ).

Now, we are going to consider the general case. As before, for $z \in$ $V\left(z_{0}\right)$, we set $f(\zeta)=g\left(\Phi_{z}(\zeta)\right)$ and we will give an estimate on $f$ instead of $g$. We will show later that it is sufficient to estimate $\frac{\partial^{k} f}{\partial \zeta_{1}^{k}}(0)$ in order to have an estimate on $\nabla^{k} g(z)$ (as $\frac{\partial}{\partial \zeta_{1}}$ is locally a transverse vector field). The proof of Theorem $B$ will be given in three steps. The first step is devoted to show that $L^{\prime k} f$ is the sum of an $(A B)$-function and a rest. The second step is devoted to give an estirnate of $\frac{\partial^{k} f}{\partial \zeta_{t}^{k}}(0)$ in terms of $F^{k} f$. This gives an estimate of $\frac{\partial^{k}}{\partial \zeta_{1}^{k}}(0)$ in terms of $L^{\prime k} f$ disregarding a rest. The third and last step is devoted to estimate this rest.

First step: We are going to gencralize Lemma 2.1 and to show that we can write locally the ficld $L^{\prime k}$ as the sum of a field with (AB)-coefficients and a rest. We need the following definition.

Definition. $\phi$ is $\mathcal{O}^{\prime}\left(\delta^{j} \tau^{k}\right)$ if $\phi$ is bounded by $C \inf \left\{1, \delta^{j} \tau^{k}\right\}$. 
Lemma 3.2. For cach $\zeta \in R(z)$ and $k \in \mathbb{N}$ and encry $\mathcal{C}^{\infty}$-function,

$$
L^{\prime k} f(\zeta)=F^{k} f(\zeta)+R^{k} f(\zeta)
$$

where

$$
F^{k} f(\zeta)=\left(\left(\sum_{\substack{t+,<m_{1} \\ t, s \geq 1}} l a_{l_{1} s}(z) \zeta_{2}^{i-1} \bar{\zeta}_{2}^{-s}\right) \frac{\partial}{\partial \zeta_{1}}-\frac{1}{2} \frac{\partial}{\partial \zeta_{2}}\right)^{k} f(\zeta)
$$

and

$$
R^{k} f(\zeta)=\sum_{1 \leq j+r \leq k} \mathcal{O}^{\prime}\left(\delta^{j} \tau^{\tau+1-k}\right) \frac{\partial^{j+r} f}{\partial \zeta_{1}^{j} \partial \zeta_{2}^{r}}(\zeta)
$$

Moreover, for every $\zeta \in R(z)$, we have

$$
F^{k} f(\zeta)=\sum_{\substack{1 \leq j+r \subseteq k \\ k+j-r \leq \leq r m j}} A_{j, r}^{(k)} \frac{\partial^{j+r} f}{\partial \zeta_{j}^{j} \partial \zeta_{2}^{r}}(\zeta)
$$

where

$$
A_{0, r}^{(k)}=0 \text { for every } r<k \text { and } A_{0, k}^{(k)}=\left(-\frac{1}{2}\right)^{k},
$$

and when $j \neq 0$, there erist some contants $C_{j, r}^{(k)}$ such that

$$
A_{j, r}^{(k)}=C_{j, r}^{(k)} \sum\left(\frac{1}{2}\right)^{(k-j)} \prod_{\beta=1}^{j}\left(\sum_{\substack{l+s \leq m, j \\ l>n_{\beta}, s>1}} \frac{l !}{\left(l-n_{\beta}\right) !} a_{l_{1}, s}(z) \zeta_{2}^{l-n_{i, j}, \bar{\zeta}_{2}}\right)
$$

where the first sum is on all the possibilities of choice of the $n_{\beta}$ which are integers such that $\sum_{1 \leq \beta \leq j} n_{\beta}=k-r$.

Proof: Let $L^{\prime}=\frac{\partial \rho}{\partial \zeta_{1}} \frac{\partial}{\partial \zeta_{2}}-\frac{\partial \rho}{\partial \zeta_{2}} \frac{\partial}{\partial \zeta_{1}}$ be the complex tangential vector field. As in the proof of Lemma 2.1, there exist some constarts $c_{j, r}$; $1 \leq j+r \leq k$, such that

$$
L^{\prime k}=\sum_{1 \leq j+r \leq k} \sum_{E_{k, j, r}} c_{j, r}\left(\prod_{i=1}^{k} \frac{\partial^{m_{i}+r_{i}} \varrho}{\partial \zeta_{1}^{m_{i}} \partial \zeta_{2}^{n_{1}}}\right) \frac{\partial^{j+r}}{\partial \zeta_{1}^{j} \partial \zeta_{2}^{r}}
$$

where $E_{k, j_{1},}$ denotes the set of couples $\left(m_{i}, n_{i}\right), i=1, \ldots, k$, which are in lexicographical order and satisfy $\sum_{i=1}^{k} m_{i}=k-j, \sum_{2=1}^{k} n_{i}=k-r^{r}$ with 
$m_{i}+n_{i} \geq 1$. Furthermore, there exists at least one integer $i_{0} \in\{1, \ldots, k\}$ such that $\left(m_{i_{0}}, n_{i_{0}}\right)=(0,1)$ or $(1,0)$.

We know that since $\varrho$ is a $\mathcal{C}^{\infty}$-function, cach coefficient of this sum is uniformly bounded on $V\left(z_{0}\right)$. Using Lemma 1.1, we also know that, for every $\zeta \in R(z)$ and every $1 \leq n \leq m-1$

$$
\begin{aligned}
& \frac{\partial^{n} \varrho}{\partial \zeta_{2}^{n}}(\zeta)=\sum_{i \geq 1} \frac{l !}{(l-n) !} a_{l, s}(z) \zeta_{2}^{i-n} \bar{\zeta}_{2}^{s}+\mathcal{O}^{\prime}\left(\delta \tau^{1-n}\right) \\
& \frac{\partial^{m} \varrho}{\partial \zeta_{2}^{m L}}(\zeta)=\mathcal{O}(\tau) \text { and } \frac{\partial \varrho}{\partial \zeta_{1}}(\zeta)=\frac{1}{2}+\mathcal{O}(\tau) .
\end{aligned}
$$

In particular, as $\sum\left|a_{l, s}(z)\right|_{\tau}(z)^{l+s} \simeq \delta(z)$ by property 6 ,

$$
\left|\frac{\partial^{n} \varrho}{\partial \zeta_{2}^{n}}\right| \leq C \min \left\{\delta \tau^{-n}, \tau\right\} \text { on } R(z) \text {, for } n=1, \ldots, m \text {. }
$$

Let us share the summation on $E_{k, j, r}$ into two parts:

$$
E_{k, j, r}=E_{k, j, r}^{\prime} \cup E_{k, j, r}^{\prime \prime}
$$

where $E_{k, j, r}^{\prime}$ is the set of couples $\left(m_{i}, n_{i}\right)$ such that either $m_{i}$ or $n_{i}$ is equal to $0, n_{i} \leq m-1$, and $m_{i} \in\{0,1\}$.

The sum on $E_{k, j, r}^{\prime}$ gives a sum of terms like

$$
\left[\left(\frac{\partial \varrho}{\partial \zeta_{1}}\right)^{k-j} \times \prod_{\beta=1}^{j}\left(\frac{\partial^{n_{\beta}} \varrho}{\partial \zeta_{2}^{n_{\beta}}}\right)\right] \frac{\partial^{j+r}}{\partial \zeta_{1}^{j} \partial \zeta_{2}^{r}} \text { with } \sum_{\beta=1}^{j} n_{\beta}=k-\tau
$$

which can be written

$$
\begin{array}{r}
{\left[\left(\frac{1}{2}\right)^{(k-j)} \prod_{\beta=1}^{j}\left(\sum_{\substack{l+\leq \leq n, n, j \\
l \geq n \beta, \alpha \geq 1}} \frac{l !}{\left(l-n_{\beta}\right) !} a_{l_{1}, i}(z) \zeta_{2}^{i-n_{\beta}} \bar{\zeta}_{2}^{s}\right)+\right.} \\
\left.+\mathcal{O}^{\prime}\left(\delta^{j} \tau^{r-k+1}\right)\right] \frac{\partial^{j+r}}{\partial \zeta_{1}^{j} \partial \zeta_{2}^{r}}
\end{array}
$$

with $\sum_{1 \leq \beta \leq j} n_{\beta}=k-r$. This gives

$$
\begin{aligned}
\sum_{j, r} \sum_{E_{k, j, r}^{\prime}} c_{j, r}\left(\prod_{i=1}^{k} \frac{\partial^{m_{i}+n_{i}} \varrho}{\partial \zeta_{1}^{m_{i}} \partial \zeta_{2}^{n_{i}}}\right) \frac{\partial^{j+r} f}{\partial \zeta_{1}^{j} \partial \zeta_{2}^{r}}= \\
=\sum_{\substack{i \leq j+r \leq k \\
k+j_{j-r} \leq m j}}\left[A_{j, r}^{(k)}+\mathcal{O}^{\prime}\left(\delta^{j} \tau^{r-k+1}\right)\right] \frac{\partial^{j+r}}{\partial \zeta_{1}^{j} \partial \zeta_{2}^{r}}= \\
\quad=F^{k} f+\sum_{1 \leq j+r \leq k} \mathcal{O}^{\prime}\left(\delta^{j} \tau^{r-k+1}\right) \frac{\partial^{j+r} f}{\partial \zeta_{1}^{j} \partial \zeta_{2}^{r}} .
\end{aligned}
$$


It remains to show that each term corring from ${E^{\prime}}_{k, j, r}^{\prime}$ is also $\mathcal{O}^{\prime}\left(\delta^{3} \tau^{r-k+1}\right)$ and, in fact, as each coefficient is bounded, it suffices to show this estimate only when $\delta^{j} \tau^{r-k+1} \leq C$.

So, let $(M, N)=\left(m_{i}, n_{i}\right)_{i=1, \ldots, k}$ fixed in ${E^{\prime}}_{k, j, r}^{\prime}$. Let $J$ be the number of $m_{i}$ which are equal to 0 . Then, $J \geq j$ since $\sum_{i} m_{i}=k-j \geq k-J$. If $J=0$ then $j=0$ and all the $m_{i}$ 's are equal to 1 , but $k-r \geq 1$ because otherwise $(M, N)$ would be in $E_{k, j, r}^{\prime}$. In this case, $\mathcal{O}^{\prime}\left(\delta^{j} \tau^{r-k+1}\right)=\mathcal{O}(1)$, so we can conclude.

Now, let $J \geq 1$. We have $m_{1}=\ldots=m_{J}=0$. We can assume that $(m+1) j>k-r$, otherwise $\mathcal{O}^{\prime}\left(\delta^{j} \tau^{r-k+1}\right)=\mathcal{O}(1)$ on $E_{k, j, r}^{\prime \prime}$ since

$$
\begin{aligned}
& \text { when } j \geq 1, \quad \delta^{j} \tau^{r-k+1} \geq C \tau^{m j+r-k+1} \geq C \tau^{1-j} \geq C, \\
& \text { and when } j=0, \quad \tau^{r-k+1} \geq C \text { since } r<k \text { on }{E^{\prime}}_{k, 0, r^{*}}^{\prime}
\end{aligned}
$$

So, let us assume that $(m+1) j>k-r$. Then, there exists at least one $n_{i} \leq m$ for some $i \leq J$. Let us denote by $K$ the number of $n_{2} \leq m$ for $i \leq J$; so $n_{1}, \ldots, n_{K} \leq m$. Then, the corresponding coefficient is bounded in absolute value by

$$
C\left|\prod_{i=1}^{K} \frac{\partial^{n_{i}} \varrho}{\partial \zeta_{2}^{n_{i}}}\right|
$$

which is clearly bounded by

$$
C \delta^{K} \tau^{-\Sigma_{i=1}^{K} n_{i}} \leq C \delta^{K} \tau^{(J-K)(m+1)-\Sigma_{i=1}^{l} n_{i}}
$$

since $\sum_{i=1}^{K} n_{i} \leq \sum_{i=1}^{J} n_{i}-(m+1)(J-K)$. So, as $\tau^{m} \leq \delta$, this coefficient is bounded by $C \delta^{J} \tau^{(J-K)-\Sigma_{i=1}^{J} n_{i}}$.

Now, as $J \geq j$ and $\sum_{i=1}^{J} n_{i} \leq k-r$, we obtain the good estimate when $J>j$, or $J-K \geq 1$, or when $\sum_{i=1}^{J} n_{i} \leq k-r-1$.

It remains to consider the case when $K=J=j$ and $\sum_{i=1}^{j} n_{i}=k-r$ and we can ever assume that $n_{i}=m$ for at least one $i \leq j$, since $(M, N) \notin E_{k, j, r^{*}}^{\prime}$ Let us remind that $\left|\frac{\partial^{m} \rho}{\partial \zeta_{2}^{m}}\right|=\mathcal{O}(\tau)$ and denote by $L$ the number of $n_{i} \leq n-1$. Then; by assumption, $j-L=J-L \geq 1$ and the corresponding coefficient is bounded by

$$
C \delta^{L} \tau^{-\sum_{i=1^{n}}^{L} n_{i}+(j-L)} \leq \delta^{L} \tau^{m(j-L)+r-\hbar+(j-L)}
$$

since $\sum_{i=1}^{L} n_{i}=k-\tau-m(j-L)$. So, we obtain that this coefficient is bounded by $C \delta^{j} \tau^{r-k+j-L} \leq C \delta^{j} \tau^{r-k+1}$.

This gives the lemma. 
Second step: Now, we want to estimate $\left|\frac{\partial^{k} f}{\partial \zeta_{1}^{k}}(0)\right|$ in terms of $F^{k} f$. We are going to use Lemma 3.2 and in particular, the peculiar form of the function $F^{k} f$ which is $(A B)$ because $f$ is holomorphic.

We recall that, by property 6 of the function $\tau$, wc have, for each $z \in V\left(z_{0}\right)$

$$
\sum_{\substack{l+s \leq m \\ l, s \leq 1}}\left|a_{l, s}(z)\right| \tau(z)^{l+s} \simeq \delta(z) \quad \text { with constants independent of } z \text {. }
$$

We deduce that, if $\left(\varepsilon_{l, s}\right)_{\substack{t+, \leq m \\ l, s \geq 1}}$ is a sequence of reals in $] 0,1[$, which will be choser later on, there exists at least one of the couples $(l, s) ; l+s \leq$ $m, l, s \geq 1$ such that $\left|a_{l, s}(z)\right| \tau(z)^{l+s} \geq \varepsilon_{l, s} \delta(z)$. So, it suffices to estimatie $\left|a_{l, s}(z)\right|^{k} \tau(z)^{(l+s-1) k}\left|\frac{\partial^{k} f}{\partial \zeta_{1}^{k}(0)}\right|$ when $\left|a_{l, s}(z)\right| \tau(z)^{l+s} \geq \varepsilon_{l, s} \delta(z)$ in order to obtain an estimate on $\left(\frac{\delta(z)}{\tau(z)}\right)^{k}\left|\frac{\partial^{k} f}{\partial \zeta_{1}^{k}}(0)\right|$.

We obscrve that

$$
\frac{\partial^{(m-1) k} F^{k} f}{\partial \bar{\zeta}^{(m-1) k}}(0)=a_{1, m-1}^{k}(z) \frac{\partial^{k} f}{\partial \zeta_{1}^{k}}(0)
$$

and we deduce, by the (AB)-functions' properties, that, when $\left|a_{1, m-1}(z)\right|$ $\tau(z)^{m} \geq \varepsilon_{1, m-1} \delta(z)$,

$$
\left(\varepsilon_{1, m-1}\right)^{p k}\left(\frac{\delta(z)}{\tau(z)}\right)^{p k}\left|\frac{\partial^{k} f}{\partial \zeta_{1}^{k}}(0)\right|^{p} \leq C M \operatorname{ean}^{R(z)}\left(\mid F^{k} f^{p}\right) .
$$

Now, if $\left|a_{1, m-1}(z)\right| \tau(z)^{m}<\varepsilon_{1, m-1} \delta(z)$, we have to find another couple $(L, S)$ such that $\left|a_{L, S}(z)\right| \tau(z)^{L+S} \geq \varepsilon_{L, S} \delta(z)$ and for which a correct estimate holds.

We introduce the following total order relation: let $l, s, L, S$ be four integers such that $l, s, L, S \geq 1$ and $L+S \leq m, l+s \leq m$; we write that $(l, s)<(L, S)$ if $s>S$ or else, if $l<L$ and $s=S$.

In the following lemma, we choose a sequence $\left(\varepsilon_{l, s}\right)_{\substack{1+s \leq m \\ i+s \geq 1}}$ so that, if the couple $(L, S)$ is the first one (in the order $<$ ) for which $\left|a_{L, S}(z)\right| \tau(z)^{L+S} \geq$ $\varepsilon_{L, S} \delta(z)$, then, $\left|a_{L, S}(z)\right|^{k p} \tau(z)^{(L+S-1) k p}\left|\frac{\partial^{k} f}{\partial \zeta_{1}^{k}}(0)\right|^{p}$ can be bounded by the mean-valuc of $\left|L^{\prime k} f\right|^{p}$, disregarding a rest.

Lemma 3.3. For every $\varepsilon>0$, every $p>0$ and cvery $k \in \mathbb{N}$, there exists a sequence $\left(\varepsilon_{t, s}\right)_{\substack{t, a \geq 1 \\ t+a \leq m}}$ of reals in $] 0,1[$ such that, if we assume that

$$
\begin{array}{r}
\left|a_{l, s}(z)\right| \tau(z)^{l+s}<\varepsilon_{l, s} \delta(z) \text { for every }(l, s)<(L, S) \text { with } \\
L, S \geq 1, L+S \leq m \text { flxed, }
\end{array}
$$


then, there exists a constant $C$, independent of $\varepsilon$, such that

$$
\begin{aligned}
& \left|a_{L, S}(z)\right|^{p k} r(z)^{(L+S-1) p k}\left|\frac{\partial^{k} f}{\partial \zeta_{1}^{k}}(0)\right|^{p} \leq C M e a l^{n(z)}\left(\left|F^{k} f\right|^{p}\right)+ \\
& +C \sum_{\substack{0 \leq j \leq k \\
1 \leq r+j \leq k(m-1)}} \min \left\{\varepsilon^{p} \varepsilon_{L, S}^{p k}\left(\delta^{j} \tau^{r-k}\right)^{p}, \tau^{p k}\right\}\left|\frac{\partial^{j+r} f}{\partial \zeta_{1}^{j} \partial \zeta_{2}^{r}}\right|^{p}
\end{aligned}
$$

Remark. In particular, if in addition $\left|a_{L, S}(z)\right| \tau(z)^{\ell+S} \geq \varepsilon_{L, S} \delta(z)$, then, there exists a constant $C(\varepsilon)$ such that

$$
\begin{aligned}
\left(\frac{\delta(z)}{\tau(z)}\right)^{p k} & \left|\frac{\partial^{k} f}{\partial \zeta_{1}^{k}}(0)\right|^{p} \leq C \operatorname{Mean}^{R(z)}\left(C(\varepsilon)\left|F^{k} f\right|^{p}\right)+ \\
& +\sum_{\substack{0 \leq j \leq k \\
1 \leq \cdots,+\leq k(m-1)}} \operatorname{mirl}\left\{C \varepsilon^{p}\left(\delta^{j} \tau^{r-k}\right)^{p}, C(\varepsilon) \tau^{p k}\right\}\left|\frac{\partial^{j+r} f}{\partial \zeta_{1}^{j} \partial \zeta_{2}^{r}}\right|^{p}(0)
\end{aligned}
$$

where $C(\varepsilon)$ is chosen so that $C(\varepsilon) \geq \frac{1}{\varepsilon_{L, S}^{p k}}$ for any $L, S \geq 1, L+S \leq m$.

Proof of lemina 3.3: Using formula (3.2) of Lemma 3.2, we first observe that the term $\left(\alpha_{L, S}(z)\right)^{k} \frac{\partial^{k} f}{\partial \zeta_{1}^{k}}(0)$ appears in $\frac{\partial^{k(S+L-1)} F^{k} f}{\partial \bar{\zeta}_{2}{ }^{k S} \partial \zeta_{2}^{k(L+1)}}(0)$. If we can estimate the other terms appearing in this expression, we can next use

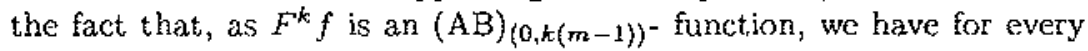
$p>0$

$$
\tau(x)^{p k(S+i,-1)}\left|\frac{\partial^{k(S+L-1)} F^{k} f}{\partial \bar{\zeta}_{2}^{k S} \partial \zeta_{2}^{k(L-1)}}(0)\right|^{p} \leq C M e a n h^{R(z)}\left(\left|F^{k} f\right|^{p}\right) .
$$

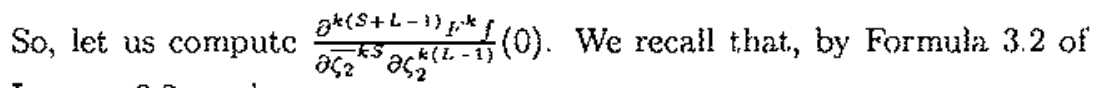
Lemma 3.2 , we have

$$
F^{k} f(\zeta)=\sum_{\substack{l \leq j+r \leq k \\ k+1, \ldots \leq m j}} A_{j, r}^{(k)} \frac{\partial^{j+r} f}{\partial \zeta_{1}^{j} \partial \zeta_{2}^{r}}(\zeta)
$$

with

$$
A_{j, r}^{(k)}=C_{j, r}^{(k)} \sum\left(\frac{1}{2}\right)^{(k-j)} \prod_{\beta=1}^{j}\left(\sum_{\substack{1+s \leq m \\ l \geq n_{\beta}, s \geq 1}} \frac{l !}{\left(l-n_{\beta}\right) !} a_{i, s}(z) \zeta_{2}^{i-n_{\beta}} \bar{\zeta}^{s}\right)
$$


where the first, sum is on all the possibilities of choice of the $n_{\beta}$ which are integers such that $\sum_{1 \leq \beta \leq j} n_{\beta}=k-r$.

So, we can see that $\frac{\partial^{k(S+L-1)} F^{*} J}{\partial \bar{\zeta}_{2}^{k S} \partial \zeta_{2}^{k(L-1)}}(0)$ is the sum, mp to constant coefficients, of the following terms

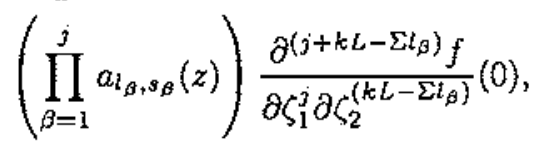

where $\sum_{1 \leq \beta \leq j} s_{\beta}=k S$ and $k L-\sum_{1 \leq \beta \leq j} l_{\beta}+j \geq 1,0 \leq j \leq k$. We will denote such a term by

where $r=k L-\sum l_{\beta}$.

$$
b_{j, r}(z) \frac{\partial^{j+r} f}{\partial \zeta_{1}^{j} \partial \zeta_{2}^{r}}(0)
$$

So, when $\left(l_{\beta}, s_{\beta}\right)=(L, S)$ for each $\beta$, we obtain $\left(a_{\ell, s}(z)\right)^{k} \frac{\partial^{k} f}{\partial \zeta_{1}^{k}}(0)$. For the extra terms, among all the $\left(l_{\beta}, s_{\beta}\right)$ 's different, from $(L, S)$, there exists at least one of them for which $\left(l_{\beta}, s_{\beta}\right) \bar{Z}(L, S)$ : either all the $s_{\beta}$ 's are not equal to $S$, so one of them is necessarly strictly bigger than $S$ by conditions $\sum s_{\beta}=k S$ and $j \leq k$; otherwise, all the $s_{\beta}$ 's are equal to $S$ and so, $j$ is equal to $k$ and necessarly there exists $l_{\beta}<L$ since $\sum l_{\beta} \leq k L$ and $\left(l_{\beta}, s_{\beta}\right) \neq(L, S)$ for at least one $\beta$.

For such a $\left(l_{\beta}, s_{\beta}\right)$, by assumption, $\left|a_{l_{\beta, s_{\beta}}}(z)\right| \tau(z)^{l_{\beta}+s_{\beta}} \leq \bar{l}_{l_{\beta}, s_{\beta}} \delta(z)$ while for every $\left(l_{\beta}, s_{\beta}\right),\left|a_{l_{\beta}, s_{\beta}}(z)\right| r(z)^{l_{\beta}+s_{\beta}} \leq \delta(z)$. So, since clearly $\left|b_{j, r}(z)\right| \leq C$, by

$\left|b_{j, r}(z)\right| \tau(z)^{k(L+S-1)}\left|\frac{\partial^{j+r} f}{\partial \zeta_{1}^{j} \partial \zeta_{2}^{r}}(0)\right|$ is bounded by $C \tau(z)^{k}\left|\frac{\partial^{j+r} f}{\partial \zeta_{1}^{j} \partial \zeta_{2}^{r}}(0)\right|$ and

$$
C\left(\sum_{\left(l_{\beta}, s_{\theta}\right)<(L, S)} \varepsilon_{l_{\beta}, s_{\beta}}\right) \delta(z)^{j} \tau(z)^{r-k}\left|\frac{\partial^{j+r} f}{\partial \zeta_{1}^{j} \partial \zeta_{2}^{r}}(0)\right|
$$

Now, for $\varepsilon>0$ fixed, it suffices to choose a sequence $\left(\varepsilon_{l, s}\right)_{\substack{+, x \leq m \\ l, x \geq 1}}$ of reals in $] 0$, I such that, for every $(L, S), L, S \geq 1, L+S \leq m$

$$
\varepsilon_{L, S}^{k} \geq \frac{1}{\varepsilon} \sum_{(l, s)<(L, S)} \varepsilon_{l, s}
$$

This can be done easily. From these, we deduce that the extra terms give a contribution bounded by

$$
C \sum_{\substack{0 \leq \leq \leq r \\ 1 \leq r+j \leq k<m-1)}} \min \left\{\varepsilon^{p} \varepsilon_{L, S}^{p k}\left(\delta^{j} \tau^{r-k}\right)^{p}, \tau^{p k}\right\}\left|\frac{\partial^{j+r} f}{\partial \zeta_{1}^{j} \partial \zeta_{2}^{r}}\right|^{p}(0) .
$$

We deduce from the preceding lemma the following corollary. 
Corollary 3.4. For every $\varepsilon>0$, every $p>0$ and every $k \in \mathbb{N}$, there exists a constant $C(\varepsilon)$ such that

$$
\begin{gathered}
\delta(z)^{p k}\left|\frac{\partial^{k} f}{\partial \zeta_{1}^{h}}(0)\right|^{p} \leq \operatorname{Mean}{ }^{R(z)}\left(C(\varepsilon) \tau(z)^{p k}\left(\left|L^{\prime k} f\right|^{p}+\left|R^{k} f\right|^{p}\right)\right)+ \\
+\sum_{\substack{0 \leq j \leq k \\
1<r+j \leq k(m-1)}} \min \left\{C \varepsilon^{p}\left(\delta^{j} \tau^{r}\right)^{p}, C(\varepsilon) \tau^{2 p k}\right\}\left|\frac{\partial^{j+r} f}{\partial \zeta_{1}^{j} \partial \zeta_{2}^{r}}\right|^{p}(0) .
\end{gathered}
$$

Proof: We have observed that, if $\left|a_{1, m-1}(z)\right| \tau(z)^{m} \geq \varepsilon_{1, m_{-1}-1} \delta(z)$, then

$$
\begin{aligned}
\left(\varepsilon_{1, m-1}\right)^{p k}\left(\frac{\delta(z)}{\tau(z)}\right)^{p k}\left|\frac{\partial^{k} f}{\partial \zeta_{1}^{k}}(0)\right|^{p} & \leq C M \operatorname{can}^{n(z)}\left(\left|F^{k} f\right|^{p}\right) \leq \\
& \leq C \operatorname{Mean}{ }^{n(z)}\left(\left|L^{\prime k} f\right|^{p}+\left|R^{k} f\right|^{p}\right)
\end{aligned}
$$

and by definition, thore exists no $(l, s), l+s \leq m, l, s \geq 1$ such that $(l, s)<(1, m-1)$. So, in this case, we find Corollary 3.4 with $\varepsilon=0$; otherwise, we apply Lemma 3.3 to the first couple $(L, S), L+S \leq m, L, S \geq 1$ for the order $\overline{<}$ sueh that $\left\{a_{l, L}(z) \mid \tau(z)^{l+S} \geq \varepsilon_{L, S} \delta(z)\right.$. This gives the result.

Third step: Theorem B will follow from corollary 3.4 as soon as we have given a bound to the rernaining t,crms

$$
\begin{aligned}
M \operatorname{ean}^{R(x)} & \left(C(\varepsilon)\left|R^{k} f\right|^{p}\right)+ \\
& +\sum_{\substack{0 \leq j \leq k \\
1 \leq n \leq j \leq k n-1)}} \min \left\{C \varepsilon^{p}\left(\delta^{j} \tau^{r}\right)^{p}, C(\varepsilon) \tau^{2 p k}\right\}\left|\frac{\partial^{j+r} f}{\partial \zeta_{1}^{j} \partial \zeta_{2}^{r}}\right|^{p}
\end{aligned}
$$

which are majorized by

$$
(*)=\operatorname{Mean}{ }^{R(z)}\left(\sum_{\substack{0 \leq j \leq k \\ 1 \leq r+j \leq k(m-1)}} \mathcal{O}_{r, 3}^{k}(\varepsilon)\left|\frac{\partial^{j+r} f}{\partial \zeta_{1}^{j} \partial \zeta_{2}^{r}}\right|^{y}\right)
$$

where we recall that the $\mathcal{O}_{r_{j}}^{k}(\epsilon)$ are defined by

$$
\mathcal{O}_{r, j}^{k}(\varepsilon)(z)=\left\{\begin{array}{l}
C(\varepsilon) \delta^{j+1}(z) \tau^{r}(z)+C \varepsilon \delta^{j}(z) \tau^{r}(z) \text { if } \delta^{j}(z) \tau^{r}(z) \leq c \tau^{k}(z) \\
C(\varepsilon) \delta^{j}(z) \tau^{r+1}(z) \text { if } c \tau^{k}(z) \leq \delta^{j}(z) \tau^{r}(z) \leq C \tau^{k-1}(z) \\
C(\varepsilon) \tau^{k}(z) \text { otherwise. }
\end{array}\right.
$$


Observe that, since $\delta^{j} \tau^{r-k+1} \geq \tau^{m j+r-k+1}$, we have

$$
\mathcal{O}_{\tau, j}^{k}(\varepsilon)(z) \leq\left\{\begin{array}{l}
C(\varepsilon) \delta^{j+1}(z) \tau^{r}(z)+C \varepsilon \delta^{j}(z) \tau^{r}(z) \\
C(\varepsilon) \tau^{k}(z) \text { when } m j+r-k<0
\end{array}\right.
$$

We are going to estimate each derivative $\frac{\partial}{\partial \zeta_{2}}$ by $L^{\prime}$, with the help of Lemma 3.2. Explicitely, we have, on $R(z)$,

$$
\left(\frac{\partial \varrho}{\partial \zeta_{1}}\right)^{r} \frac{\partial^{r}}{\partial \zeta_{2}^{r}}=L^{\prime r}+\sum_{\substack{1 \leq j+s \leq r \\ s \leq r .1}} \mathcal{O}^{\prime}\left(\delta(z)^{j} \tau(z)^{y-r}\right) \frac{\partial^{j+s}}{\partial \zeta_{1}^{j} \partial \zeta_{2}^{s}}
$$

and we can assume that $\frac{\partial \rho}{\partial \zeta_{1}} \neq 0$ on $R(z)$. This allows us to obtain an estimate on each derivative $\left|\frac{\partial^{j * r} f}{\partial \zeta_{1}^{j} \partial \zeta_{2}^{\gamma}}\right|$ in terms of $\left|L^{\prime r} \frac{\partial^{j} f}{\partial \zeta_{1}^{j}}\right|$ and of derivatives either of order strictly less than $j+r$, or of order with respect to $\zeta_{2}$ strictly less than $r$.

Applying successively this estimate and using the fact that $\mathcal{O}_{r, j}^{r}(\varepsilon) \geq$ $\mathcal{O}_{r^{\prime}, j^{\prime}}^{r}(\varepsilon)$ when $r \leq r^{\prime}$ and $j \leq j^{\prime}$, we obtain that.

$$
(*) \leq C \operatorname{Mean}^{\prime \prime(z)}\left(\sum_{1 \leq r+j \leq k(m-1)} \mathcal{O}_{r, 3}^{k}(\varepsilon)\left|L^{\prime r} \frac{\partial^{j} f}{\partial \zeta_{1}^{j}}\right|^{\nu}\right) .
$$

So, after a change of coordinates,

$$
\delta(z)^{p k}\left|M_{z}^{k} g(z)\right|^{p j} \leq M e a n^{Q(z)}\left(C(\varepsilon) \tau(z)^{p k}\left|\nabla_{T}^{k} g\right|^{p}+R e s t^{k}(\varepsilon)^{p}\right),
$$

where $M_{k}$ is the vector ficld $M_{z}=\left(\frac{\partial \tau}{\partial z_{1}}(z)\right)^{-1} \frac{\partial}{\partial z_{3}}$ which is transverse at $z$.

Now, we need the following lemma.

Lemma 3.5. Let $r, j \in \mathbb{N}$. There exists a constant $C$ such that, for every holomorphic function $g$ in $\Omega$, every $z \in V\left(z_{0}\right) \cap \Omega$, we have

$$
\left|\nabla_{T}^{r} \nabla^{j} g\right|(z) \leq C M e a n^{Q(z)}\left(\left|\nabla_{T}^{r} \nabla^{j} g\right|+\operatorname{Rest}^{r}\left(\nabla^{j} g\right)\right),
$$

where $\operatorname{Rest}^{r}(u)=\sum_{1 \leq s+t \leq r} \mathcal{O}^{\prime}\left(\delta^{s} \tau^{t-r+1}\right)\left|\nabla_{t^{\prime}}^{t} \nabla^{s} u\right|$.

Proof: We will just give the main idcas of the proof in $\mathbb{C}^{2}$.

It is sufficient to prove the result for $j=0$. For general $j$, we write the inequality for $\nabla^{j} g$ instead of $g$. As before, we set $f=g \circ \Phi_{z}$ and we will 
give an estimate on $L^{\prime r} f(0)$ in order to obtain an estimate on $\nabla_{\gamma^{\prime}, 3}^{r}(z)$. By lemma 3.2, we have

$$
\begin{aligned}
\left|L^{\prime r} f(0)\right| & \leq\left|F^{r} f(0)\right|+\left|R^{r} f(0)\right| \leq \\
& \leq C M \operatorname{Mean}{ }^{R(z)}\left(\left|F^{r} f\right|+\left|R^{r} f\right|\right) \leq \\
& \leq C \operatorname{Mean}^{R(z)}\left(\left|L^{\prime r} f\right|+\left|R^{r} f\right|\right) .
\end{aligned}
$$

So, the lemma follows, estimating $R^{r} f$ as beforc.

Now, in order to conclude for Theorem $B$, it is cnough to remark that there exists a constant $C$ such that, for $z \in V\left(z_{0}\right)$

$$
\left|\nabla^{k} g(z)\right| \leq C\left(\left|M_{z}^{k} g(z)\right|+\sum_{\substack{j, r=k \\ r \geq 1}}\left|\nabla_{T^{\prime}}^{r} \nabla^{j} g(z)\right|+\sum_{1 \leq j \leq k-1}\left|\nabla^{j} g(z)\right|\right) .
$$

By Lemma 3.5, wo are able to estimate each $\left|\nabla_{T}^{r} \nabla^{j} g(z)\right|$ by its meanvalue on $Q(z)$, disregarding a rest. This allows us to sec that

$$
\delta(z)^{k} \sum_{\substack{j+r:=k \\ r \geq 1}}\left|\nabla_{T}^{r} \nabla^{j} g(z)\right|+\delta(z)^{k} \sum_{1 \leq \mathbb{E}-1}\left|\nabla^{j} g(z)\right| \leq C M \operatorname{can}^{Q(z)}\left(\operatorname{Rest} t^{k}(\varepsilon)\right) .
$$

We will assume now that $\Omega$ is bounded. So, the estimates of Theorems $\mathrm{A}$ and $\mathrm{B}$ are uniformly valid on $\Omega \cap U$, where $U$ is a neighborhood of $\partial \Omega$ sufficiently small so that the projection on $\partial \Omega$ and the function $\tau($ ) are well defined on $\Omega \cap U$.

In the following, $K$ will denote a compact in $\Omega$ containing the cornplement of $\Omega \cap U$ and $\delta_{0}$ will denote the real sup $\{\delta(z) ; z \in \Omega \cap U\}$.

\section{4. $L^{p}$-results}

First of all, we are going to give sorne tools necessary for the proof of Theorem C.

\subsection{A Whitney Lemma.}

Recall that there exists a constant $C$ such that, if $z^{\prime} \in Q_{\delta}(z)$ then $Q_{\delta}\left(z^{\prime}\right) \subset Q_{C r}(z)$. We denote by $Q^{\prime}$ the C-uple of $Q$.

Lemma 4.1. There exists a set of points $z_{k} \in \Omega \cap U, k \in \mathbb{N}$, satisfying the following properties:

- the family $\left\{Q\left(z_{k}\right) ; k \in \mathbb{N}\right\}$ covers $\Omega \cap U$. 
- each $Q^{\prime \prime}\left(z_{k}\right)\left(C\right.$-uple of $\left.Q^{\prime}\left(z_{k}\right)\right)$ is contained in $\Omega \cap W$ (where $W$ is a neighborhood of $\partial \Omega$ containing $U)$.

- there exists an integer $N$, which depends only on $\Omega$, such that no point in $\Omega \cap W$ belongs to more than $N Q^{\prime \prime}\left(z_{k}\right), k \in \mathbb{N}$.

We choose $V$ a neighborhood of $\partial \Omega$ containing $U$ and strictly included in $W$ such that all of the $Q^{\prime}\left(z_{k}\right)$ are contained in $\Omega \cap V$. A fortiori, no point of $\Omega \cap V$ belongs to more than $N$ of the sets $Q^{\prime}\left(z_{k}\right), k \in \mathbb{N}$.

Proof: Let us consider the sets $\Omega_{k}=\left\{z \in \mathbb{C}^{n} ; C^{-k} \leq \delta(z) \leq C^{-k+1}\right\}$ for $k \in\left\{k_{0}, . .,+\infty\right\}$, with $k_{0}=-\log _{C} \delta_{0}+1$ (chosen such that $\Omega \cap U=$ $\cup_{k=k_{0}}^{+\infty} \Omega_{k}, C$ is the preceding constant $\left.>1\right)$.

$Q_{\varepsilon}(z)$ contains a ball around $z$ of fixed radius $\varepsilon^{\prime}>0$, independently of $z$. It follows that wo can choose a set of points $z_{j}^{(k)} \in \Omega_{k}$ such that

$$
\Omega_{k} \subset U_{j} Q_{c C^{-k}}\left(z_{j}^{(k)}\right) \text { and } z_{j}^{(k)} \notin Q_{c C^{-k-1}}\left(z_{l}^{(k)}\right) \text { when } l \neq j
$$

Moreover, w: can choose $c$ sufficicntly small such that, for each $j, Q_{c c^{-k+2}}\left(z_{j}^{(k)}\right) \subset \overline{\Omega_{k}}$, where

$$
\overline{\Omega_{k}}=\left\{z \in \mathbb{C}^{n} ; C^{-k-2} \leq \delta(z) \leq C^{-k+3}\right\}
$$

Then, it is usual to see that no point in $\overline{\Omega_{k}}$ belongs to more than $N^{\prime}$ of the $Q_{0} \zeta^{-k+2}$, where $N^{\prime}$ is independent of $k$ (see $[\mathbf{C} \& \mathbf{W}]$ or Lemma 3.3 in [C] for instance).

We repeat this construction for each $k \in\left\{k_{0}, \ldots,+\infty\right\}$. It is clear that the family wc obtain covers $\Omega \cap U$. Now, let $z \in \cup_{j, k} Q_{c C^{-k+2}}\left(z_{j}^{(k)}\right)$. It is obvious that there exists a unique $k \in\left\{k_{0}, \ldots,+\infty\right\}$ such that $z \in \Omega_{k}$. Then, $z \in \cup_{k-2 \leq j \leq k+2} \overline{\Omega_{j}}$ and so, we conclude that $z$ does not belong to more than $N$ of the $Q_{c C^{-k+2}}$. At last, since $\delta\left(z_{j}^{(k)}\right) \simeq C^{-k}$, the sets constructed are cquivalent to the $Q\left(z_{3}^{(k)}\right)$ and the kmma is therefore proved.

\subsection{Continuity of the Mean-value Operator Mean ${ }^{2}$.}

Lemma 4.2. For every $\alpha, \beta, \gamma \in \mathbb{R}$, cvery $0<\eta<1$, the meanvalue operator Mean ${ }^{Q}$ is bounded from $L^{1}\left(\Omega \cap V, \delta^{\alpha}(\delta+\eta)^{\beta} \tau^{\gamma} d V\right)$ to $L^{1}\left(\Omega \cap U, \delta^{\alpha}(\delta+\eta)^{\beta} \tau^{\gamma} d V\right)$. Moreover the norm is independent of $\eta$.

Proof: As $\delta(.) \simeq \delta(z), \delta()+.\eta \simeq \delta(z)+\eta$ independently of $\eta$ and $\tau(., \delta().) \simeq \tau(z, \delta(z))$ on $Q(z)$, it is sufficient to prove the continuity from 
$L^{1}(\Omega \cap V, d V)$ to $L^{1}(\Omega \cap U, d V)$. This follows easily from the preceding Whitney Lemma and from Jensen's Inequality.

$$
\begin{aligned}
\int_{\Omega \cap U}\left(\operatorname{Mean}^{Q(z)}(u)\right) & d V(z) \leq \\
& \leq \sum_{k} \int_{Q\left(z_{k}\right)} \frac{1}{|Q(z)|} \int_{Q(z)}|u(\zeta)| d V(\zeta) d V(z) \leq \\
& \leq C \sum_{k} \int_{Q\left(z_{k}\right)} \frac{1}{\left|Q^{\prime}\left(z_{k}\right)\right|} \int_{Q^{\prime}\left(z_{k}\right)}|u(\zeta)| d V(\zeta) d V(z) \leq \\
& \leq C \sum_{k} \int_{Q^{\prime}\left(z_{k}\right)}|u(\zeta)| d V(\zeta) \leq \\
& \leq C \int_{\Omega \cap V}|u| d V .
\end{aligned}
$$

\subsection{Hardy Inequalities.}

Let us denote by $\frac{\partial}{\partial \nu}$ the normal derivative which is given by the field $\nu=\operatorname{Re} N$ where $N$ is the complex normal vector field $N=\sum_{j=1}^{n} \frac{\partial r}{\partial z_{z}} \frac{\partial}{\partial z_{j}}$. We have chosen $U$ so that the function $\mathcal{F}_{s}: \partial \Omega \rightarrow \bar{\Omega} \cap U ; 0 \leq s<s_{0}$, given by

$$
\mathcal{F}_{s}(z)=\exp \left(s \frac{\partial}{\partial \nu}\right)(z), \text { for } z \in \partial \Omega
$$

gives a diffeomorphism from $\partial \Omega \times\left[0, s_{0} \mid\right.$ onto $\bar{\Omega} \cap U$. Moreover, $s \simeq$ $\operatorname{dist}\left(\mathcal{F}_{s}(z), \partial \Omega\right)$. So, for every $z \in \Omega \cap U$, there exist. $\left.s \in\right] 0, s_{0}[$ and $z^{\prime} \in \partial \Omega$ such that $z=\mathcal{F}_{s}\left(z^{\prime}\right)$. We will use the following notation.

$$
\begin{aligned}
\left(|u|^{p}\right)^{\star}(z)= & \left\{\left.u\right|^{p}(z) \text { if } p \geq 1=\right. \\
= & \sup \left\{\left|u\left(\mathcal{F}_{l}\left(z^{\prime}\right)\right)\right|^{p} ;\right. \\
& t \in\left[s, s_{0} \mid\right\} \text { if } 0<p<1 \text { and } z=\mathcal{F}_{s}\left(z^{\prime}\right) \in \Omega \cap U= \\
= & 0 \text { if } 0<p<1 \text { and } z \notin \Omega \cap U .
\end{aligned}
$$

Then, we will use the following Hardy type Inequality.

Proposition 4.3. Let $k \in \mathbb{N}, p>0$. For every $\alpha, \beta, \gamma \in \mathbb{R}$ with $\gamma \geq 0, \alpha+\frac{\gamma}{m}>-1$ and $\alpha+\beta+\frac{\gamma}{m}>-1$, there exists a constant $C$ such that, for every function $u \in \mathcal{C}^{\infty}(\Omega)$ and every $\eta>0$, we have

$$
\begin{aligned}
\int_{\Omega} \delta^{\alpha}(\delta+\eta)^{\beta} \tau^{\gamma}|u|^{p} d V \leq C\left(\int_{\Omega \cap U} \delta^{\alpha+k p}(\delta\right. & +\eta)^{\beta_{\tau} \gamma}\left(\left|\frac{\partial^{k} u}{\partial \nu^{k}}\right|^{p}\right)^{\star} d V+ \\
& \left.+\sum_{0 \leq j<k} \sup _{K}\left|\nabla^{j}{ }^{j}\right|^{p}\right)
\end{aligned}
$$


The proof of this proposition will be given with the help of two lemmas. First, we consider the case $p \geq 1$.

Lemma 4.4. Let $p \geq 1, l \in \mathbb{P}$ and $\omega$ be any positive function satisfying

$$
\omega(s) \leq C\left(\frac{s}{t}\right)^{l} \omega(t) \text { for every } s, t \text { with } 0 \leq s \leq t ;
$$

then, for every $\alpha, \beta \in \mathbb{R}$ with $\alpha+l>-1$ and $\alpha+\beta+l>-1$, there exists a constant $C$ such that, for any positive, mesurable function $u$ defined on $\mathbb{R}^{+}$, we have

$$
\int_{0}^{\infty} U(s)^{p} s^{\alpha}(1+s)^{\beta} \omega(s) d s \leq C \int_{0}^{\infty}(s u(s))^{p} s^{\alpha}(1+s)^{\beta} \omega(s) d s,
$$

where $U(s)=\int_{s}^{\infty} u(t) d t$ for $s \geq 0$.

Proof: We begin with the case $\omega=1$ and $l=0$. Without loss of generality, we can assume that $\int_{0}^{\infty}(s u(s))^{p} s^{\alpha}(1+s)^{\beta} d s<\infty$. Integrating by parts the term

$$
(*)=\int_{0}^{\infty}\left(\int_{s}^{\infty} u(t) d t\right)^{p} s^{\alpha}(1+s)^{\beta} d s
$$

we obtain

$$
\begin{aligned}
(*)= & p \int_{0}^{\infty}\left(\int_{s}^{\infty} u(t) d t\right)^{\gamma-1} u(s)\left(\int_{0}^{s} t^{\alpha}(1+t)^{\beta} d t\right) d s+ \\
& +\lim _{A \rightarrow \infty}\left[\left(\int_{s}^{\infty} u(t) d t\right)^{p}\left(\int_{0}^{s} t^{\alpha}(1+t)^{\beta} d t\right)\right]_{0}^{\Lambda} \leq \\
& \leq C\left(\int_{0}^{\infty}\left(\int_{s}^{\infty} u(t) d t\right)^{p-1} u(s) s^{\alpha+1}(1+s)^{\beta} d s+\right. \\
& \left.+\lim _{A \rightarrow \infty}\left[\left(\int_{s}^{\infty} u(t) d t\right)^{p} s^{\alpha+1}(1+s)^{\beta}\right]_{0}^{\Lambda}\right), \leq(1)+(2)
\end{aligned}
$$

since $\int_{0}^{s} t^{\alpha}(1+t)^{\beta} d t \simeq\left(s^{\alpha+1}(1+s)^{\beta}\right)$ for every $s \geq 0$, as it can be seen easily.

By Hölder's Inequality

$$
\begin{aligned}
(1) \leq C\left(\int_{0}^{\infty}\left(\int_{s}^{\infty} u(t) d t\right)^{p} s^{\alpha}(1+s)^{\beta} d s\right)^{1-1 / p} & \\
& \left(\int_{0}^{\infty}(s u(s))^{p} s^{\alpha}(1+s)^{\beta} d s\right)^{1 / p},
\end{aligned}
$$


and, for every $s \geq 0$

$$
\begin{array}{r}
U(s)^{p} \leq\left(\int_{0}^{\infty}(t u(t))^{p_{t}^{\alpha}}(1+t)^{\beta} d t\right)\left(s^{\alpha+1}(1+s)^{\beta}\right)^{-1} \\
\text { so, }(2) \leq \int_{0}^{\infty}(t u(t))^{p} t^{\alpha}(1+t)^{\beta} d t .
\end{array}
$$

This allows to conclude when $\omega=1$. For general $\omega$, it suffices to use the assumption on $\omega$ in order to obtain

$$
\begin{aligned}
&(*)=\int_{0}^{\infty}\left(\int_{s}^{\infty} u(t) d t\right)^{p} s^{\alpha}(1+s)^{\beta} \omega(s) d s \leq \\
& \leq \int_{0}^{\infty}\left(\int_{s}^{\infty}\left(\frac{\omega(t)}{t^{l}}\right)^{1 / p} u(t) d t\right)^{p} s^{\alpha+l}(1+s)^{\beta} d s
\end{aligned}
$$

and to conclude by the preceding result.

When $0<p<1$, there is no such inequality for general $u$. But, we can prove the following lemma.

Lemma 4.5. Let $0<p<1, l \in \mathbb{R}$ and $\omega$ be any positive function satisfying

$$
\omega(s) \leq C\left(\frac{s}{t}\right)^{l} \omega(t) \text { for every } s, t \text { with } 0 \leq s \leq t
$$

then, for cvery $\alpha, \beta \in \mathbb{B}$ with $\alpha+l>-1$ and $\alpha+\beta+l>-1$, there exists a constant $C$ such that, for any decreasing positive function $u$ on $\mathbb{R}^{+}$, we have

$$
\int_{0}^{\infty} U(s)^{p} s^{\alpha}(1+s)^{\beta} \omega(s) d s \leq C \int_{0}^{\infty}(s u(s))^{p} s^{\alpha}(1+s)^{\beta} \omega(s) d s
$$

where $U(s)=\int_{s}^{\infty} u(t) d t$ for $s \geq 0$

Proof: We set $(* *)=\int_{0}^{\infty}\left(\int_{s}^{\infty} u(t) d t\right)^{p} s^{\alpha}(1+s)^{\beta} \omega(s) d s$; then wa write

$$
\begin{aligned}
(* *) & \leq \sum_{j=-\infty}^{\infty} \sum_{k=j}^{\infty} u\left(2^{k}\right)^{p} 2^{k p} \int_{2^{j-1}}^{2^{j}} s^{\alpha}(1+s)^{\beta} \omega(s) d s \leq \\
& \leq C \sum_{j=-\infty}^{\infty} \sum_{k=j}^{\infty} u\left(2^{k}\right)^{p} \frac{2^{k p}}{\left(2^{j}\right)^{l}} \omega\left(2^{j}\right) \int_{2^{j \cdots 1}}^{2^{j}} s^{\alpha+l}(1+s)^{\beta} d s
\end{aligned}
$$


by assurnption on $\omega$.

So, we have

$$
\begin{aligned}
(* *) & \leq C \sum_{k=-\infty}^{\infty} u\left(2^{k}\right)^{p} 2^{k p} \sum_{j=-\infty}^{k} \omega\left(2^{j}\right)\left(2^{j}\right)^{\alpha+1}\left(1+2^{j}\right)^{\beta} \leq \\
& \leq C \sum_{k=-\infty}^{\infty} u\left(2^{k}\right)^{p} 2^{k p} \omega\left(2^{k}\right) \sum_{j=-\infty}^{k}\left(\frac{2^{j}}{2^{k}}\right)^{l}\left(2^{j}\right)^{\alpha+1}\left(1+2^{j}\right)^{\beta} \leq \\
& \leq C \sum_{k=-\infty}^{\infty} u\left(2^{k}\right)^{p} 2^{k p} \omega\left(2^{k}\right)\left(2^{k}\right)^{\alpha+1}\left(1+2^{k}\right)^{\beta} \leq \\
& \leq C \int_{0}^{\infty} u(s)^{p} s^{\alpha+p}(1+s)^{\beta} \omega(s) d s .
\end{aligned}
$$

Now, we are able to prove Proposition 4.3.

First, let us observe that, by homogeneity, we carn replace $(1+s)$ by $(\eta+s)$ in the preceding lemmas (without changing the constant in the inequality). Then, by properties 2 and 4 of the function $\tau$, we see that there exists a constant $C$ such that, for cvery $z \in \partial \Omega$, we have

$$
\tau\left(\mathcal{F}_{s}(z), s\right) \leq C\left(\frac{s}{t}\right)^{1 / m} \tau\left(\mathcal{F}_{t}(z), t\right) \text { for } 0 \leq s \leq t \leq s_{0} .
$$

(This is bccause $\tau\left(\mathcal{F}_{s}(z), s\right) \leq\left(\frac{s}{l}\right)^{1 / m} \tau\left(\mathcal{F}_{l}(z), t\right)$ by property 4 , and $\tau\left(\mathcal{F}_{s}(z), t\right) \simeq \tau\left(\mathcal{F}_{l}(z), t\right)$ by property 2 , sincc $\mathcal{F}_{s}(z) \in Q\left(\mathcal{F}_{l}(z)\right)$.) So, the preceding lemrnas are valid with

$$
\omega(s)=\left\{\begin{array}{l}
\tau^{\gamma}\left(\mathcal{F}_{s}(z), s\right) \text { if } s \leq s_{D} \\
s^{\gamma / m} \text { otherwise, }
\end{array}\right.
$$

for every $z \in \partial \Omega$, with a constant independert. of $z$.

Thus, we apply the preceding lemmas successively to the function

$$
U\left(s_{1}\right)=\int_{s_{1}}^{\infty} \ldots \int_{s_{k}}^{\infty}\left[\left|\frac{d^{k} u}{d s^{k}}\left(\mathcal{F}_{s}(z)\right)\right|\right]^{\star} d s d s_{k} \ldots d s_{2}
$$

where we recall that

$$
\begin{aligned}
{\left[\left|\frac{d^{k} u}{d s^{k}}\left(\mathcal{F}_{s}(z)\right)\right|\right]=} & \sup \left\{\left|\frac{d^{k} u}{d s^{k}}\left(\mathcal{F}_{r}(z)\right)\right| ; s \leq r<s_{0}\right\} \\
& \text { if } 0<p<1 \text { and } 0<s<s_{0}= \\
= & \left|\frac{d^{k} u}{d s^{k}}\left(\mathcal{F}_{s}(z)\right)\right| \text { if } p \geq 1 \text { and } 0<s<s_{0}= \\
= & 0 \text { if } s \geq s_{0} .
\end{aligned}
$$

(At each step, the function is decreasing wher $0<p<1$ ). We conclude by integration over $\partial \Omega$. 


\subsection{Auxiliary Lemmas.}

We have introduced in Proposition 4.3 the $\star$-operator. We will necd (for $0<p<1$ ) a sort of continuity of this operator in $p$-norms for a certain class of functions. We begin with the simpler result which gives the continuity for $(\mathrm{AB})$-functions.

Lemma 4.6. Let $0<p<1, \gamma \geq 0, \alpha, \beta \in \mathbb{B}$ with $\alpha+\frac{\gamma}{m_{2}} \geq 0$ and $\alpha+\beta+\frac{\gamma}{m} \geq 0$. There exists a constant $C$ such that, for every (AB)-function $F$ in $\Omega$ and every $\eta>0$, we have

$$
\int_{\Omega \cap U} \delta^{\alpha}(\eta+\delta)^{\beta} \tau^{\gamma}\left(|F|^{p}\right)^{\star} d V \leq C\left(\int_{\Omega \cap V} \delta^{\alpha}(\eta+\delta)^{\beta} \tau^{\gamma}|F|^{p} d V\right) .
$$

Proof: The proof is similar to the one given in [St] or in [B\&S] for harmonic functions.

We cover $\Omega \cap U$ as in Lemrna 4.1 by the family $\left\{Q\left(z_{2}\right) ; i \in I\right\}$ and we denote by $\chi_{i}$ the characteristic function of $Q\left(z_{i}\right) ; i \in I$. Then

$$
(*)=\int_{\Omega \cap U} \delta^{c x}(\eta+\delta)^{\beta} \tau^{\gamma}\left(|F|^{p}\right)^{*} d V \leq \sum_{i} \int \delta^{\alpha}(\eta+\delta)^{\beta} \tau^{\gamma}\left(\chi_{i}|F|^{p}\right)^{*} d V .
$$

But, $\left(\chi_{i} \mid F^{p}\right)^{*}$ is supported in the set

$$
E_{i}=\left\{z \in \Omega ; \exists s>0 \text { such that } z-s \nu_{2} \in Q\left(z_{i}\right)\right\}
$$

which is of measure less than $C\left|Q\left(z_{i}\right)\right|$.

On $E_{i}$, we have:

$$
\delta(z)^{\alpha}(\eta+\delta(z))^{\beta} \tau^{\gamma}(z, \delta(z)) \leq C\left(\delta\left(z_{i}\right)^{\alpha}\left(\eta+\delta\left(z_{i}\right)\right)^{\beta} \tau^{\gamma}\left(z_{i}, \delta\left(z_{i}\right)\right)\right) .
$$

This last assertion follows from the fact that, by assurnption on the indices, the function $x \rightarrow x^{r+\frac{\gamma}{n n}}(\eta+x)^{\beta}$ is increasing for $x \geq 0$ (as $\alpha+\frac{\gamma}{m} \geq 0$ and $\alpha+\beta+\frac{\gamma}{m} \geq 0$ ) and from property 2 and 4 of the finction $\tau$ which imply that, for every $z \in E_{i}$, we have

$$
\begin{aligned}
\tau(z, \delta(z)) \leq & C\left(\frac{\delta(z)}{\delta(z)+s}\right)^{1 / m} \tau(z, \delta(z)+s) \leq \\
\leq & C\left(\frac{\delta(z)}{\delta(z)+s}\right)^{1 / m} \tau\left(z-s \nu_{z}, \delta(z)+s\right) \\
& \text { since } z-s \nu_{z} \in Q_{\delta(z)+s}(z) \leq \\
\leq & C\left(\frac{\delta(z)}{\delta\left(z_{i}\right)}\right)^{1 / m} \tau\left(z_{i}, \delta\left(z_{i}\right)\right) .
\end{aligned}
$$


So, we have

$$
\left(\chi_{i}|F|^{p}\right)^{\star} \leq \sup _{Q\left(z_{i}\right)}\left\{|F|^{p}\right\}
$$

Then, applying the (AB)-functions' propertics, we obtain

$$
\sup _{Q\left(z_{i}\right)}\left\{|F|^{p}\right\} \leq \frac{C}{\left|Q^{\prime}\left(z_{i}\right)\right|} \int_{Q^{\prime}\left(z_{i}\right)}|F|^{p} d V .
$$

So,

$$
\begin{aligned}
(*)= & \int_{\operatorname{\Omega n} U} \delta^{\alpha}(\eta+\delta)^{\beta} \tau^{\gamma}\left(|F|^{p}\right)^{*} d V \leq \\
& \leq C \sum_{i} \frac{\delta\left(z_{i}\right)^{\alpha}\left(\eta+\delta\left(z_{i}\right)\right)^{\beta} \tau^{\gamma}\left(z_{i}\right)\left|Q\left(z_{i}\right)\right|}{\left|Q^{\prime}\left(z_{i}\right)\right|} \int_{Q^{\prime}\left(z_{i}\right)}|F|^{p} d V \leq \\
& \leq C \int_{\Omega \cap V} \delta^{c \alpha}\left(\eta_{\gamma}+\delta\right)^{\beta} \tau^{\gamma}|F|^{p} d V
\end{aligned}
$$

Now, let us give a more difficult lemma.

Lemma 4.7. Let $0<p<1, t \geq 0, \alpha, \beta \in \mathbb{R}$ and $k \in \mathbb{N}$ such that $\alpha+\frac{t+k_{p}}{m} \geq 0, \alpha+\beta+\frac{t+k_{p}}{m} \geq 0$ and $\alpha+\beta+\frac{t}{m}>-1, \alpha+\frac{t}{m}>-1$; then there exists a constant $C$ such that, for every $\eta>0$ and every $g$ holomorphic in $\Omega$, we have

$$
\begin{aligned}
\int_{\Omega \cap U} \delta^{\alpha}(\eta+\delta)^{\beta} \tau^{l+k p}\left(\left|\nabla_{T}^{k} g\right|^{p}\right)^{*} d V \leq C\left(\int_{\Omega \cap W} \delta^{\alpha}(\eta+\delta)^{\beta} \tau^{l+k p}\left|\nabla_{\gamma}^{k} g\right|^{p} d V+\right. \\
\left.+\int_{\Omega \cap W} \delta^{\alpha+k p}(\eta+\delta)^{\beta} \tau^{t}\left|\nabla^{k} g\right|^{p} d V+\int_{K}|g|^{\dot{p}} d V\right)
\end{aligned}
$$

Remark. In addition, we can choose the constart in front of the second term on the right hand side of the inequality as small as $\varepsilon$ and $\delta_{0}$.

Proof: As in the preceding proof, we cover $\Omega \cap U$ by the family $\left\{Q\left(z_{i}\right)\right.$, $i \in I\}$ and we denote by $\chi_{i}$ the characteristic function of $Q\left(z_{i}\right), i \in I$. Then,

$$
\begin{aligned}
&(*)=\int_{\Omega \cap U} \delta^{\alpha}(\eta+\delta)^{\beta} \tau^{\ell+k p}\left(\left|\nabla_{1}^{k} g\right|^{p}\right)^{*} d V \leq \\
& \leq \sum_{i} \int \delta^{\alpha}(\eta+\delta)^{\beta} \tau^{\ell+k_{p}}\left(\chi_{i}\left|\nabla_{T}^{k} g\right|^{p}\right)^{*} d V
\end{aligned}
$$


But, as before, $\left(\chi_{i}\left|\nabla_{T}^{k} g\right|^{p}\right)^{\star}$ has a support contained in the set.

$$
E_{i}=\left\{z \in \Omega ; \exists s>0 \text { such that } z-s \nu_{z} \in Q\left(z_{i}\right)\right\}
$$

which satisfies, in particular, for every $z \in E_{i}$,

$$
\delta(z)^{\alpha}(\eta+\delta(z))^{\beta} \tau^{l+k p}(z, \delta(z)) \leq C\left(\delta\left(z_{i}\right)^{\alpha}\left(\eta+\delta\left(z_{i}\right)\right)^{\beta} \tau^{t+k p}\left(z_{i}, \delta\left(z_{i}\right)\right)\right)
$$

(by assumption on the indices).

So,

$$
\begin{aligned}
(*) & =\int_{\Omega \cap U} \delta^{\alpha}(\eta+\delta)^{\beta} \tau^{l+k p}\left(\left|\nabla_{T}^{k} g\right|^{p}\right)^{\star} d V \leq \\
& \leq C \sum_{i} \delta\left(z_{i}\right)^{\alpha}\left(\eta+\delta\left(z_{i}\right)\right)^{\beta} \tau^{t+k p}\left(z_{i}, \delta\left(z_{i}\right)\right)\left|Q\left(z_{i}\right)\right| \sup _{Q\left(z_{2}\right)}\left\{\left|\nabla_{\eta^{\prime} g}^{k}\right|^{p}\right\} .
\end{aligned}
$$

Now, on $Q\left(z_{i}\right)$, each component of $\nabla_{T}^{k} g$ is equal, after a change of coordinate $z=\Phi_{z_{i}}(\zeta)$, to the sum of an $(A B)$-function and a rest. Let $F^{k} f$ be the vector of these (AB)-functions. Wo have, for $f=g \circ \Phi_{z_{i}}$

$$
\begin{aligned}
\sup _{Q\left(z_{i}\right)}\left\{\left|\nabla_{J}^{k} g\right|^{p}\right\} & \leq \sup _{R\left(z_{i}\right)}\left\{\left|F^{k} f\right|^{p}+\left|R^{k} f\right|^{p}\right\} \leq \\
& \leq \frac{1}{\left|R^{\prime}\left(z_{i}\right)\right|} \int_{R\left(z_{i}^{\prime}\right)}\left|F^{k} f\right|^{p} d V+\sup _{R\left(z_{i}\right)}\left\{\mid R^{k} f\left[^{p}\right\} \leq\right. \\
& \leq \frac{1}{\left|Q^{\prime}\left(z_{i}\right)\right|} \int_{Q^{\prime}\left(z_{i}\right)}\left|\nabla_{T}^{k} g\right|^{p} d V+\frac{1}{\left|Q^{\prime}\left(z_{i}\right)\right|} \int_{Q^{\prime}\left(z_{i}\right)}\left|R e s t^{k} g\right|^{p} d V+ \\
& +\sup _{Q\left(z_{i}\right)}\left\{\left|R e s t^{k} g\right|^{p}\right\}
\end{aligned}
$$

where $\left|R e s t^{k} g\right| \leq \sum_{r=0}^{k-1} \sum_{1 \leq j+r \leq k} \mathcal{O}^{\prime}\left(\delta^{j} \tau^{r+1-k}\right)\left|\nabla_{j}^{r} . \nabla^{j} g\right|$. Now, we just, have to estimate the rest. By the direct estimates, we have

$$
\left|\operatorname{Rest}^{k} g\right| \leq C \operatorname{Mean}^{Q}\left(\sum_{r=0}^{k-1} \sum_{j \leq j+r \leq k}\left(\frac{\mathcal{O}^{\prime}\left(\delta^{i} \tau^{r+1-k}\right)}{\tau^{r}}\right)\left|\nabla^{j} g\right|\right),
$$

and since for $z \in Q\left(z_{i}\right), Q(z) \subset Q^{\prime}\left(z_{i}\right)$, we obtain

$$
\begin{aligned}
\sup _{Q\left(z_{i}\right)}\left\{\left|\operatorname{Res} t^{k} g\right|^{p}\right\} & \leq C \sup _{Q^{\prime}\left(z_{i}\right)}\left\{\sum_{j=0}^{k}\left(\frac{\mathcal{O}^{\prime}\left(\delta^{j} \tau^{\tau+1-k}\right)}{\tau^{r}}\right)^{p}\left|\nabla^{j} g\right|^{p}\right\} \leq \\
& \leq C \sup _{Q^{\prime}\left(z_{i}\right)}\left\{\sum_{j=0}^{k}\left(\frac{\mathcal{O}\left(\delta^{j}\right)}{\tau^{k}}\right)^{p}\left|\nabla^{j} g\right|^{p}\right\} \leq \\
& \leq \frac{C}{\left|Q^{\prime \prime}\left(z_{i}\right)\right|} \int_{Q^{\prime \prime}\left(z_{i}\right)} \sum_{j=0}^{k} \frac{\delta^{j p}\left|\nabla^{j} g\right|^{p}}{\tau^{k p}} d V .
\end{aligned}
$$


So, by Lemma 4.1

$$
\begin{gathered}
(* *)=\sum_{i} \delta\left(z_{i}\right)^{\alpha}\left(\eta+\delta\left(z_{i}\right)\right)^{\beta} \tau\left(z_{i}, \delta\left(z_{i}\right)\right)^{i+k p}\left|Q\left(z_{i}\right)\right| \sup _{Q\left(z_{i}\right)}\left\{\mid \text { Rest }\left.t^{k} g\right|^{p}\right\} \leq \\
\leq C \sum_{j=0}^{k} \int_{\Omega \cap W}\left|\nabla^{j} g\right|^{p} \delta^{\alpha+j p^{\prime}}(\eta+\delta)^{\beta} \tau^{t} d V \leqq \\
\leq C \int_{\Omega \cap W W}\left(\left|\nabla^{k} g\right|^{p}\right)^{\star} \delta^{\alpha+k p}(\eta+\delta)^{\beta} \tau^{t} d V \quad \text { by Hardy Inequality } \leqq \\
\leq C \int_{\Omega \Omega \cap W}\left|\nabla^{k} g\right|^{p} \delta^{\alpha+k p}(\eta+\delta)^{\beta} \tau^{t} d V+\int_{K}|g|^{p} d V \quad \text { by Lemma 4.6. }
\end{gathered}
$$

The other term of the rest is estimated in the same way.

4.5 Proof of the $L^{p}$-result of the Auxiliary Theorem.

We recall what we are going to prows the following result.

\section{Auxiliary Theorem.}

Let $\Omega$ be a bounded $\mathcal{C}^{\infty}$ domain of fintite type $m$ in $\mathbb{C}^{n}$. There exists a compoct set $K \subset \Omega$ such that, for every $p>0$, every $k \in \mathbb{N}$ and $q, t \in \mathbb{R}$ with $q+\frac{t}{m}>-1$ and $t \geq 0$,

$$
\int_{\Omega}|g|^{p} \delta^{q} \tau^{t} d V \text { and } \int_{\Omega}\left|\nabla^{k} g\right|^{p} \delta^{q+p k} \tau^{t} d V
$$

are equivalent, modulo an error of $f_{K}|g|^{p} d V$, for $g$ holornorphic in $\Omega$.

First, it is easy to see that, by the mean-value property of holomorphic functions and the continuity of the meant-value operator (Lemma 4.2), we have

$$
\int_{\Omega}\left|\nabla^{k} g\right|^{p} \delta^{\eta+k p} \tau^{t} d V \leq C\left(\int_{\Omega}|g|^{p} \delta^{q} \tau^{t} d V+\int_{K}|g|^{p} d V\right) .
$$

The converse follows from Hardy Inequality (proposition 4.3) and Lemma 4.6

$$
\begin{aligned}
& \int_{\Omega 2}|g|^{p} \delta^{\prime \eta} \tau^{i} d V \leq C\left(\int_{\Omega \Omega U U} \delta^{y+s y} \tau^{l}\left(\left|\nabla^{s} y\right|^{p}\right)^{*} d V+\int_{K}|g|^{p} d V\right) \leq \\
& \leq C\left(\int_{\Omega} \delta^{a+s p} \tau^{\ell}\left|\nabla^{s} g\right|^{p} d V+\int_{K}|g|^{p} d V\right)
\end{aligned}
$$

if $s$ is sufficiently large, when $p<1$, in order to be able to apply the auxiliary Lemma 4.6. This gives the result if we can take $s=k$, otherwise, $s>k$ and the preceding estimate gives the result.

Now, we are able to prove Thcorem $\mathrm{C}$ with the help of Theorems $\mathrm{A}$ and $\mathrm{B}$ and all the preceding results. Namely 


\section{Theorem $\mathrm{C}$.}

Let $\Omega$ be a bounded $\mathcal{C}^{\infty}$-domain of finite type on in $\mathbb{C}^{n}$. There exists a compact set $K \subset \Omega$ such that, for every $p>0 q, t \in \mathbb{R}$ and $k, l, r \in \mathbb{N}$ with $q+p r+\frac{i}{m}>-1, q+p l+\frac{p k+t}{m}>-1$ and $t \geq 0$

$$
\begin{aligned}
& \int_{\Omega}\left|\nabla_{T}^{k} \nabla^{l} g\right|^{p} \delta^{q+p l} \tau^{k p+t} d V \\
& \int_{\Omega}\left|\nabla^{l} \nabla_{T}^{k} g\right|^{p} \delta^{g+p l} \tau^{k p+t} d V \text { and } \\
& \int_{\Omega}\left|\nabla^{r} g\right|^{p} \delta^{q+p r} \tau^{t} d V
\end{aligned}
$$

are equivalent, modulo an error of $\int_{K}|g|^{p} d V$, for $g$ holomorphic in $\Omega$.

4.6 First part of the proof of Theorem C: Direct Estimates.

We arc going to prove in this paragraph that, when $q+p l+(t+k p) / m>$ -1 and $q+p r+t / m>-1$

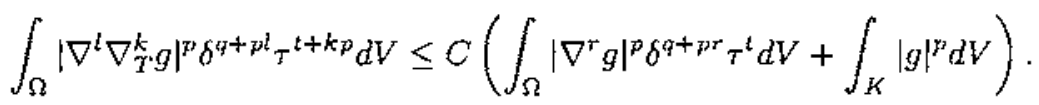

We begin with the case $l=0$. The general case follows easily from the Auxiliary Theorem and Lemma 2.2.

By Hardy Inequality (proposition 4.3), we have:

$$
\begin{aligned}
(*)= & \int_{\Omega}\left|\nabla_{T}^{k} g\right|^{p} \delta^{q} \tau^{t+k p} d V \leq \\
\leq & C\left(\int_{\Omega \Omega \cap U}\left(\left(\left|\nabla^{s} \nabla_{T}^{k} g\right|^{p}\right)^{\star} \delta^{g+s p} \tau^{l+k p} d V+\int_{K}|g|^{p} d V\right) \leq\right. \\
& \leq C\left(\int_{\Omega \cap U}\left(\left|\nabla_{T}^{k} \nabla^{s} g\right|^{p}\right)^{\star} \delta^{q+s p} \tau^{\ell+k p} d V+\right. \\
& \left.+\sum_{r=0}^{k-1} \sum_{j=1}^{s} \int_{\Omega \cap U}\left(\left|\nabla_{T}^{r} \nabla^{j} g\right|^{p}\right)^{\star} \delta^{g+s p} \tau^{t+k p} d V+\int_{K}|g|^{p} d V\right),
\end{aligned}
$$

by Lernma 2.2 . We choose $s$ sufficiently large when $p<1$ in order to be able to apply the auxiliary Lemma 4.7 . So, by lemma 4.7

$$
\begin{aligned}
(*) \leq & C\left(\int_{\Omega \cap W}\left|\nabla_{T}^{k} \nabla^{s} g\right|^{p} \delta^{q+s p} \tau^{t+k p} d V+\right. \\
& +\sum_{r=0}^{k-1} \sum_{j=1}^{s} \int_{\Omega \cap W}\left|\nabla_{T}^{r} \nabla^{j} g\right|^{p} \delta^{q+s p} \tau^{z+k p} d V+ \\
& \left.+\sum_{r=0}^{k} \sum_{j=1}^{s} \int_{\Omega \cap W}\left|\nabla^{r+j} g\right|^{p} \delta^{a+(r+s) p} \tau^{l+(k-r) p} d V+\int_{K}|g|^{p} d V\right) .
\end{aligned}
$$


Then, we apply the first part of Theorem A and the $L^{1}$-continuity of the mean-value operator (Lemma 4.2) in order to obtain

$$
\begin{aligned}
(*) \leq & C\left(\int_{\Omega \cap W}\left|\nabla^{s} g\right|^{p} \delta^{q+s p} \tau^{\iota} d V+\right. \\
& +\sum_{r=0}^{k-1} \sum_{j=1}^{s} \int_{\Omega \cap W}\left|\nabla^{j} g\right|^{p} \delta^{\eta+s p} \gamma^{\ell+(k-r) p} d V+ \\
& \left.+\sum_{r=0}^{k} \sum_{j=1}^{s} \int_{\Omega \cap W}\left|\nabla^{r+j} g\right|^{p} \delta^{q+(r+s) p} \tau^{\ell+(k-r) p} d V+\int_{K}|g|^{p} d V\right)
\end{aligned}
$$

Then applying Auxiliary Theorem and the fact that $\delta(.) \leq C \delta_{0}$ on $\Omega \cap W$, we obtain that

$$
\begin{aligned}
\int_{\Omega}\left|\nabla_{\gamma^{k}}^{k} g\right|^{p} \delta^{y} \tau^{l+k p} d V & \leq C\left(\int_{\Omega}\left|\nabla^{s} g\right|^{p} \delta^{g+s p} \tau^{t} d V+\int_{K}|g|^{p} d V\right) \leq \\
& \leq \leq C\left(\int_{\Omega}\left|\nabla^{r} g\right|^{p} \delta^{q+\tau p} \tau^{t} d V+\int_{K}|g|^{p} d V\right)
\end{aligned}
$$

\subsection{Second part: Converse Estimates.}

We are going to prove now that, when $q+p l+(t+k p) / m>-1$ and $q+p r+t / m>-1$

$$
\int_{\Omega}\left|\nabla^{r} g\right|^{p} \delta^{q+p r} \tau^{l} d V \leq C\left(\int_{\Omega}\left|\nabla^{l} \nabla_{T}^{k} g\right|^{p} \delta^{s+p^{l}} \tau^{l+k p} d V+\int_{K}|g|^{p} d V\right) .
$$

Wc assume that $l=0$ and $r=k$. The gcneral case follows easily by the Auxiliary Theorern. To simplify, we will assume also that $p \geq 1$. When $p<1$, the proof follows the same lines except that we have to apply sufficiently the Hardy Inequality in order to be able to apply the auxiliary lemmas.

The prof will be given in two parts.

First part: an a priori estimate.

Lemma 4.8. Let $p \geq 1, k \in \mathbb{N}, q, t \in \mathbb{R}$ with $t \geq 0$ and $q+(p k+$ t) $/ m>-1$, then there exists a constant $C$ such that, for every $\eta>0$ and every $g$ holomorphic in $\Omega$ satisfying $g \in \mathcal{C}^{\infty}(\bar{\Omega})$, we have

$$
\int_{\Omega}\left|\nabla^{k} g\right|^{p} \delta^{p k}(\eta+\delta)^{q} \tau^{t} d V \leq C\left(\int_{\Omega}\left|\nabla_{T}^{k} g\right|^{p}(\eta+\delta)^{q} \tau^{t+k p} d V+\int_{K}|g|^{p} d V\right)
$$


Proof: For each $\varepsilon>0$, every $\eta>0$, we have

$$
\begin{aligned}
\int_{\Omega}\left|\nabla^{k} g\right|^{p} \delta^{p k}(\eta+\delta)^{q} \tau^{\ell} d V \leq C\left(\int_{\Omega}\left|\nabla_{T}^{k} g\right|^{p}(\eta+\delta)^{q} \tau^{\ell+k p} d V+\right. \\
\left.+\int_{\Omega \cap U} \operatorname{Rest^{k}}(\varepsilon)^{p}(\eta+\delta)^{q} \tau^{\imath} d V+\int_{K}|g|^{p} d V\right)
\end{aligned}
$$

by the converse estimates of Theorem B. So, it suffices to estimate the remaining term.

$$
(*)=\int_{\Omega \cap U} \operatorname{Rest}^{k}(\varepsilon)^{p}(\eta+\delta)^{q} \tau^{t} d V
$$

First, we observe that $\mathcal{O}_{r, j}^{k}(\varepsilon)$ is always bounded by $\left(C \varepsilon+C(\varepsilon) \delta_{0}^{1 / m}\right) \delta^{j} \tau^{r}$ and is bounded by $C \tau^{k}$ when $j+(r-k) / m<0$. So we obtain

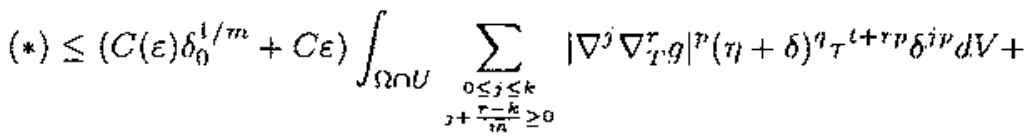

$$
\begin{aligned}
& +C(\varepsilon) \int_{\Omega \cap U} \sum_{\substack{0 \leq j \leq k \\
j+\frac{\tau-k}{m}<0}}\left|\nabla^{j} \nabla_{\eta}^{r} g\right|^{p}(\eta+\delta)^{q} \tau^{l+k^{\prime}} d V
\end{aligned}
$$

Then, we can apply Hardy Inequality to each term in order to obtain

$$
\begin{aligned}
(*) \leq\left(C(\varepsilon) \delta_{0}^{1 / m}+C \varepsilon\right) \int_{\Omega \cap U} \sum_{r}\left|\nabla^{k} \nabla_{T}^{r} g\right|^{p}(\eta+\delta)^{q} \tau^{l+r p} \delta^{k p} d V+ \\
+C(\varepsilon) \int_{\Omega \cap U} \sum_{\substack{0 \leq j \leq k \\
j 1 \frac{r-k}{m}<0}}\left|\nabla^{k} \nabla_{T}^{r} g\right|^{p}(\eta+\delta)^{a} \tau^{l+k p} \delta^{(k-j) p} d V
\end{aligned}
$$

which gives with Theorem A

$$
\begin{aligned}
(*) \leq & \left(C(\varepsilon) \delta_{0}^{1 / m}+C \varepsilon\right) \int_{\Omega \cap U}\left|\nabla^{k} g\right|^{p}(\eta+\delta)^{q} \tau^{l} \delta^{k p} d V+ \\
& +C(\varepsilon) \int_{\Omega \cap U} \sum_{\substack{a \leq j \leq k \\
j+\frac{\sum_{j}-k}{m}<o}}\left|\nabla^{k} g\right|^{p}(\eta+\delta)^{q} \tau^{l+(k-r) p} \delta^{(k-j) p} d V \leq \\
& \leq\left(C(\varepsilon) \delta_{0}^{1 / m}+C \varepsilon\right) \int_{\Omega \cap U}\left|\nabla^{k} g\right|^{p}(\eta+\delta)^{q} \tau^{l} \delta^{k p} d V
\end{aligned}
$$

as $m j+r-k+1 \leq 0$ in the sccond term (so that $\delta^{-j} \tau^{k-r-1} \leq$ $\tau^{-m j+k-r-1} \leq C$ ). 
So, the result of the lernma follows by choosing $\varepsilon$ and $\delta_{0}$ sufficiently small (reducing $\Omega \cap U$ if necessary).

Second part: use of the a priori estimate

We denote by $\Omega_{\varepsilon}=\left\{z \in \mathbb{C}^{n} ; \quad r(z)<-\varepsilon\right\}$ for earh $\varepsilon>0$. We apply the preceding lemma in $\Omega_{E}$, to $g$ holomorphic in $\Omega$ and $\mathcal{C}^{\infty}\left(\overline{\Omega_{E}}\right)$ for every $\varepsilon>0$. Then there exists a constant $C$ independent of $\varepsilon$ and $\eta$ such that

$$
\int_{\Omega_{\varepsilon}}\left|\nabla^{k} g\right|^{p} \delta_{\varepsilon}^{p k}\left(\eta+\delta_{\varepsilon}\right)^{q} \tau_{\varepsilon}^{\ell} d V \leq C\left(\int_{\Omega_{\varepsilon}}\left|\nabla_{T}^{k} g\right|^{p}\left(\eta+\delta_{\varepsilon}\right)^{q} \tau_{\varepsilon}^{\ell+k p} d V+\int_{K}|g|^{p} d V\right) .
$$

Then, for $\eta=\varepsilon$, we obtain, as $\delta \simeq \delta_{\varepsilon}+\varepsilon$, for every $\varepsilon>0$

$$
\int_{\Omega_{\varepsilon}}\left|\nabla^{k} g\right|^{p} \delta_{E}^{p k} \delta^{q} \tau_{\varepsilon}^{t} d V \leq C\left(\int_{\Omega_{\varepsilon}}\left|\nabla_{T}^{k} g\right|^{p} \delta^{g} \tau_{\varepsilon}^{l+k p} d V+\int_{K}|g|^{p} d V\right) .
$$

So, it suffices now to let $\varepsilon \rightarrow 0$ in order to conclude by the monotone convergence Theorem as $\delta_{\varepsilon} \rightarrow \delta$ and $\tau_{E} \rightarrow \tau$ in increasing, $t+k p \geq 0$, $t \geq 0$ and $p k \geq 0$.

\section{5. $L^{\infty}$-results: proof of the second part of the Auxiliary Theorem and proof of Theorem D}

As the proofs follow the same line of the proof of the first part of the Auxiliary Theorem and of Theorem $\mathrm{C}$ respectively and are simpler, we will just give, for the reader's converience, the anatog of the Hardy Inequality which is the following lemma.

Lemma 5.1. Let $\alpha \in \mathbb{R}$ and $\beta \geq 0$. There exists a constant $C$ such that, for every function $u \in \mathcal{C}^{\infty}(\Omega)$, we have, for every $\mu>0$

$$
\begin{aligned}
& \sup _{\Omega}\left\{\mathcal{O}^{\prime}\left(\delta^{\alpha} \tau^{\beta}\right)|u|\right\} \leq \\
& \quad \leq C\left(\sup _{\Omega}\left\{\mathcal{O}^{\prime}\left(\delta^{\alpha+1} \tau^{\beta}\right)\left|\frac{\partial u}{\partial \nu}\right|\right\}+\sup _{K}|u|\right) \text { if } \frac{-\beta}{\alpha} \notin\{2, \ldots, m\} . \\
& \sup _{\Omega}\left\{\delta^{\prime \prime} \mathcal{O}^{\prime}\left(\delta^{\alpha} \tau^{\beta}\right)|u|\right\} \leq \\
& \quad \leq C\left(\sup _{\Omega}\left\{\mathcal{O}^{\prime}\left(\delta^{\alpha+1} \tau^{\beta}\right)\left|\frac{\partial u}{\partial \nu}\right|\right\}+\operatorname{sip}_{K}|u|\right) \text { if } \frac{-\beta}{\alpha} \in\{2, . ., m\} .
\end{aligned}
$$

Proof: As $u \in \mathcal{C}^{\infty}(\Omega)$, it is sufficient to prove the preceding result on $\Omega \cap U$. 
Without loss of generality, we can assume that. $M=\sup _{\Omega}\left\{\mathcal{O}^{\prime}\left(\delta^{\alpha+1} \tau^{\beta}\right)\right.$ $\left.\left|\frac{\partial u}{\partial \nu}\right|\right\}<\infty$. Then, we have

$$
\begin{aligned}
& |u(\zeta)|=\left|\int_{0}^{\delta_{0}} \frac{d}{d s} u\left(\zeta-s \nu_{\zeta}\right) d s+u\left(\zeta-\delta_{0} \nu_{\zeta}\right)\right| \leq \\
& \leq M \int_{0}^{\delta_{0}} \frac{d s}{\mathcal{O}^{\prime}\left((\delta(\zeta)+s)^{(x+1} \tau^{\beta}\left(\zeta-s \nu_{\zeta}, \delta(\zeta)+s\right)\right)}+\sup _{K}|u| \leq \\
& \leq M \int_{0}^{\delta_{0}} \frac{d s}{(\delta(\zeta)+s)^{\alpha+1} \tau^{\beta}\left(\zeta-s \nu_{\zeta}, \delta(\zeta)+s\right)}+M C \int_{0}^{\delta_{0}} d s+\sup _{K}|u|
\end{aligned}
$$

Let us estimate

$$
(*)=\int_{0}^{\delta_{0}} \frac{d s}{(\delta(\zeta)+s)^{\alpha+1} \tau^{\beta}\left(\zeta-s \nu_{\zeta}, \delta(\zeta)+s\right)} .
$$

As $\zeta-s \nu_{\zeta} \in Q_{\delta(\zeta)+s}(\zeta)$, we have, by the second property of $\tau$, that,

$$
\tau\left(\zeta-s \nu_{\zeta}, \delta(\zeta)+s\right) \simeq \tau(\zeta, \delta(\zeta)+s)
$$

Then

$$
\begin{aligned}
(*) & \leq \int_{0}^{\delta_{0}} \frac{d s}{(\delta(\zeta)+s)^{\alpha+1} \tau^{\beta}(\zeta, \delta(\zeta)+s)} \leq \\
& \leq \int_{0}^{\delta_{0}} \max _{2 \leq l \leq m} \frac{A_{l}(\zeta)^{\beta / l}}{(\delta(\zeta)+s)^{\alpha+1+\beta / l}} d s \leq \\
& \leq C \operatorname{mix}_{2 \leq l \leq m} \int_{0}^{\delta_{0}} \frac{A_{l}(\zeta)^{\beta / l}}{(\delta(\zeta)+s)^{\alpha+1+\beta / h}} d s
\end{aligned}
$$

since $\max _{2 \leq l \leq m} \simeq \sum_{2 \leq l \leq m}$.

But,

$$
\int_{0}^{\delta_{0}} \frac{A_{i}(\zeta)^{\beta / l}}{(\delta(\zeta)+s)^{\alpha+1+\beta / l}} d s \leq\left\{\begin{array}{l}
C A_{l}(\zeta)^{\beta / l} \delta(\zeta)^{-(\alpha+\beta / t)} \text { if } \alpha+\beta / l>0 \\
C \text { if } \alpha+\beta / l<0 \\
A_{l}(\zeta)^{\beta / l} \delta(\zeta)^{-(\alpha+\beta / l)} \log \left(\frac{1}{\delta(\zeta)}\right) \\
\text { if } \alpha+\beta / l=0
\end{array}\right.
$$

So,

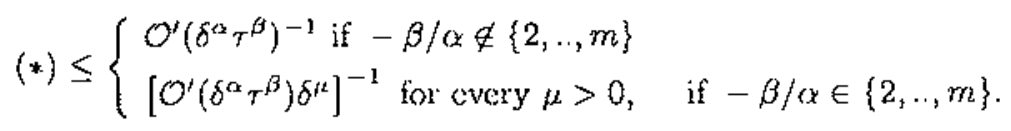




\section{Theorems $\mathbf{E}$ and $\mathbf{F}$}

We are going to give the proof of Theorem E. The proof of Theorcm $\mathrm{F}$ follows the same line.

\section{Theorem E.}

Let $\Omega$ be a bounded $\mathcal{C}^{\infty}$-domain of finite type and $s \geq 0$. There exist a compact set $K \subset \Omega$ and a constant $C>0$ such that, for every holomorphic function $g$ in $\Omega$,

$$
\int_{\Omega}\left|\nabla^{l} \nabla_{T^{k}}^{k} g\right|^{p} \delta^{p(l-s-k / m)_{T}^{k p}} d V \leq C\left(\left\|\nabla_{T}^{k} g\right\|_{W^{*, p, p}(\Omega)}^{p}+\int_{K}|g|^{p} d V\right)
$$

for $l$ integer, $p(l-s)>-1$.

Proof: Let $g$ be a holomorphic function in $\Omega$ and $s \geq 0$. First, let us remark that when $s$ is an integer, the result of Theorern $E$ follows easily from the results of Theorem $\mathrm{C}$ since, for $l \in \mathbb{N}$ with $p(l-s)>-1$

$$
\int_{\Omega}\left|\nabla^{s} \nabla_{T}^{k} g\right|^{p} \delta^{-k y / m} \tau^{k p} d V \text { and } \int_{\Omega}\left|\nabla^{l} \nabla_{T}^{k} g\right|^{p} \delta^{(l-s-k / m)} \tau^{k p} d V
$$

are equivalent, modulo an error of $\int_{K}|g|^{p} d V$; and

$$
\int_{\Omega}\left|\nabla^{s} \nabla_{T}^{k} g\right|^{p} \delta^{-k p / m} \tau^{k p} d V \leq\left\|\nabla_{T}^{k} g\right\|_{W \sim, p(\Omega)}^{p}
$$

This allows to conclude. The same remark allows the proof to be reduced to the case $s \in] 0,1[$.

So, it suffices to estimate $\int_{\Omega}\left|\nabla \nabla_{T}^{k} g\right|^{p p} \delta^{p\left(1-s-k / r^{\prime}\right)} \tau^{k p} d V$. The idea of the proof is the one used in [Bo\&Si]: it is to apply the following inequality.

For every $\delta>0$

$$
\delta^{p} \int_{B(0, \delta)}|\nabla u|^{p} d V \leq C\left(\int_{B(0,2 \delta)}|u|^{p} d V+\delta^{2 p} \int_{B(0,2 \delta)}|\Delta u|^{p} d V\right)
$$

where $C$ is independent of $\delta$.

Let us denote by $\left\{B_{j}, j \in J\right\}$ a covering of Whitney's type, where the $B_{j}$ 's are isotropic balls whose radius is proportionnal to the distance to the boundary $\delta_{j}$. Let $j \in J$ and $\zeta \in B_{j}^{\prime}$ ( $B_{j}^{\prime}$ is the ball with the same 
center as $B_{j}$ but whose radius is twice). Let us apply the preceding inequality to the function $z \rightarrow \nabla_{\gamma}^{k} g(z)-\nabla_{7}^{k} g(\zeta)$.

$$
\begin{aligned}
\delta_{j}^{p} \int_{B_{3}}\left|\nabla\left(\nabla_{T}^{k} g\right)\right|^{p} d V \leq C\left(\int_{B_{j}^{\prime}} \mid \nabla_{T}^{k} g(z)\right. & -\left.\nabla_{T}^{k} g(\zeta)\right|^{p} d V(z)+ \\
& \left.+\delta_{j}^{2 p} \int_{B_{j}^{\prime}}\left|\Delta\left(\nabla_{7}^{k} g\right)\right|^{p} d V\right) .
\end{aligned}
$$

So, integrating in the $\zeta$ variable over $B_{j}^{\prime}\left(z_{j}, \delta_{j}\right)$ and observing that, on this set, we have $|z-\zeta|<c \delta_{j}, \delta(.) \sim \delta_{j}, \tau(., \delta().) \sim \tau\left(z_{j}, \delta_{j}\right)$, we obtain

$$
\begin{aligned}
& (*)_{j}=\int_{B_{j}}\left|\nabla\left(\nabla_{T}^{k} g\right)\right|^{p} \delta^{p(1-s-k / m)} \tau^{k p} d V \leq \\
& \leq C\left(\iint_{B_{j}^{\prime} \times B_{j}^{\prime}} \frac{\left|\nabla_{T}^{k} g(z)-\nabla_{T}^{k} g(\zeta)\right|^{z}}{|z-\zeta|^{2 n+p s}} d V(z) d V(\zeta)+\right. \\
& \left.\quad+\int_{B_{j}^{\prime}}\left|\Delta\left(\nabla_{T}^{k} g\right)\right|^{p p} \delta^{p(2-s-k / \pi)} \tau^{k p p} d V\right)
\end{aligned}
$$

(since $\delta^{-k / m} \tau^{k} \leq C$ ). So, summing on $j$, we obthin

$$
\begin{aligned}
\sum_{j}(*)_{j} & \leq \int_{\Omega 2}\left|\nabla\left(\nabla_{T}^{k} g\right)\right|^{p} \delta^{p(1-s-k / m)} T^{k p} d V \leq \\
& \leq C\left(\iint_{\Omega \times \Omega \Omega} \frac{\mid \nabla_{T^{\prime} g(z)-\left.\nabla_{T}^{k} g(\zeta)\right|^{p}}^{k}}{|z-\zeta|^{2 n+p s}} d V(z) d V(\zeta)+\right. \\
& \left.++\int_{\Omega}\left|\Delta\left(\nabla_{T}^{k} g\right)\right|^{p} \delta^{p(2-s-k / m)} \tau^{k p} d V+\int_{K}|g|^{p p} d V\right) \leq \\
& \leq C\left(\left\|\nabla_{T}^{k} g\right\|_{W / p(\Omega)}^{p}+\int_{\Omega}\left|\Delta\left(\nabla_{T}^{k} g\right)\right|^{p} \delta^{p(2-s-k / m)} \tau^{k p} d V+\right. \\
& \left.+\int_{K}|g|^{p p} d V\right) .
\end{aligned}
$$

But, by the harmonicity of $g$, we have $\Delta\left(\nabla_{T}^{k} g\right)=\left[\Delta, \nabla_{?}^{k}\right] g$, so,

$$
\left|\Delta\left(\nabla_{T}^{k} g\right)\right| \leq C\left(\sum_{\substack{i \leq j \leq 2 \\ 0 \leq r \leq k-1}}\left|\nabla_{T}^{r} \nabla^{j} g\right|\right) .
$$

We have to estimate

$$
\sum_{\substack{1 \leq j \leq 2 \\ 0<r<k-1}} \int_{\Omega \Omega}\left|\nabla_{\psi}^{r} \cdot \nabla^{j} g\right|^{p} \delta^{p(2-s-k / m)} \tau^{k \cdot p} d V .
$$


But, this term is less than $\left.\int_{\Omega}\left|\nabla\left(\nabla_{1}^{k} g\right)\right|^{p} \delta^{p(1-s-k / m)}\right)_{T}^{(k+1) p} d V+\int_{K}|g|^{p} d V$ by Theorem $\mathrm{C}$. So, we obtain an a priori estimate and we conclude as in the preceding paragraph: we apply this incquality in $\Omega_{E}$ to $g$ holomorphic in $\Omega$. Wo obtain

$$
\begin{aligned}
& \int_{\Omega_{\varepsilon}}\left|\nabla \nabla_{T}^{k} g\right|^{p} \delta_{\varepsilon}^{p(1-s-k / m)} \tau_{\varepsilon}^{k p} d V \leq C\left\|\nabla_{T}^{k} g\right\|_{W N_{1} p\left(\Omega_{\varepsilon}\right)}^{p}+\int_{K}|g|^{p} d V \leq \\
& \leq C\left\|\nabla_{T}^{k} g\right\|_{W=, r(\Omega)}^{p,}+\int_{K}|g|^{\mu} d V
\end{aligned}
$$

and wo conclude by Fatou's Lemma.

Now, it suffices to apply the results of Theorem $\mathrm{C}$ and $\mathrm{D}$ to obtain the results of Corollary $B$.

Acknowledgements. I would like to thank my advisor Aline Bonami for holpful discussions and suggestions about this work.

These results have been announced in a previous paper called

"Comportement des fonctions holomorphes dans les directions complexes tangentes d'un ouvert de $\mathbb{C}^{n}$ de type fini" (sce; [G2]).

\section{References}

[A\&B] Almern, P. AND Brun , J., Maximal and Area Integral Charactorizations of Hardy-Sobolev Spaces in the Unit Ball of $\mathbb{C}^{n}$, Rev. Iberoamericana 4 (1988), 123-153.

[B] Bentrous, F., Bchavior of Holomorphic Functions Near Weakly Pseudoconvex Boundary Points, Ind. Univ. Math. J. 40, no. 3 (1991).

[B\&G] BI,OOM, T. AND GRAHAM, I, A geometric Characterization of points of type in on real submanifolds of $\mathbb{C}^{n}, J$. of Diff. Geometry 12 (1977), 171-182.

[B\&S] BOAS, H.P. AND S'rlinube, E.J., Sobolev Noms of Harmonic and Analytic Functions, Unpublished mamuscript.

[Bo\&Si\} BonnMI, A. AND SiBONy, X., Sobolev imbedding in $\mathbb{C}^{n}$ and $\bar{\partial}$-Equation, Journal of Geometric Analysis 1, no. 4 (1991).

[C] CATIJIN, D., Estirnates of Invariant Metrics on Pseudoconvex Domains of Dimension Two, Math. Zcit. 200 (1989), 429-466.

[C\&K] CIIANG, D.C. AND KRANTZ, S., Holomorphic Lipschitz functions and application to the $\bar{\partial}$-problem, Colloquium Mathernoticam LXII, no. 2 (1991), 227-256. 
[C\&W] COIPMAN, R. AND WLISS, G., "Analyse harmonique noncommutatine sur certains espaces homogènes," Lecture Notes in Math. 242, 1971.

[D] DuRen, "Theory of $H^{\gamma}$ spaces," Academic Press, New York, 1970.

[F\&S] Fornarss, J.E. ANi SIBONY, N., Construction of P.S.H functions on weakly pseudoconvex domains, Duke Math.J. 58, no. 3 (1989), I71-182.

[G\&S] Greiner, P.C. AND Stein, E.M., "Estimates for the $\bar{\partial}-N e u$ monn Problem," Princeton University Press, 1977.

[G1] GreLLisR, S., Comportement des fonctions holomorphes dans les directions complexes tangentes, $C . R$. Acad. Sci. Paris t312, série I (1991), 267-270.

[G2] GRELAIER, S, Comportement des fonctions holomorphes dans les directions complexes tangentes d'un ouvert do $\mathbb{C}^{7}$ de type fini, C. R. Acad. Sci. Paris t312, série I (1991), 777-780.

[Gr] Gilsvard, P., "Elliptic Problems in nom smooth domains," Pittman, 1985.

[H] Hormander, L., "Subelliptic Operators, in Seminat on Singularities of Solutions," Princeton University Press, 1978, pp. 127-208.

$[\mathrm{K}]$ Kolin, J.J., Boundary Behaviour of $\bar{\partial}$ on weakly psendo-convex manifolds of dimerision two, J. Diff. Geometry 6 (1972), 523-542.

[Kr1] Kraxiz, S.G., Function Theory of Several Complex Variables, John Wilcy \& sons.

[Kr2] Kn^NT\%, S.G., Finite Type Conditions and Elliptic Boundary Value Problems, , J. of Diff. Equations 34, no. 2 (1979), 239-260.

[Kr3] KR^NT\%, S.G., On a Theorem of Stein, Trans. Amer. Math. Soc. 320 , no. 2 (1990), 625-642.

[L] Licock $\wedge, E$., Estimates in Sobolev norms $\|.\|_{s, p}$ for Hammonic and Folomomphic Functions and Interpolation between Sobokv and Hölder spaces of Harmonic Functions, Studio Moth. 86 (1987), 255-271 .

[NSW] NAGei, A., STEIN, E.M. AND Wainger, S., Bonndary Behavior of finctions holomorphic in domains of finite type, Proc. Nat. Acad. Sci USA 78 (1981), 6596-6599.

[P] Pesrenson, Z., Functions which are smooth along vector ficlds, Mathematical Notes of the Academy of Sciences of the USSR 148 (1990), 683-695.

[R\&S] Rotscmuld, L.P. AND STRIN, E.M., Hypoelliptic Differential Operator and Nilpotent Groups, Acta. Math. 137 (1977), 248-315. 
[S] STeIN, E.M., "Boundary Behavior of Holonorphic Functions of Several Complex Variables," Princeton University Press, Princeton, 1972.

[St] STraube, E., Interpolation between Sobolev and between Lipschitz Spaces of Analytic Functions on Starshaped Domains, Trans. Amer. Math. Soc. 316 (1989), 653-671.

Département de Mathérratiques et d'informatique d'Orléans Universilé d'Orléans B.P. 6759 45067 Orléans Cedex 2 FRANCE

Primera versió rebuda el 2 de Setembre de 1991 , darrera versió rebuda el 17 de Febrer de 1992 